\title{
Soil moisture retrieval over agricultural fields from L- band multi-incidence and multitemporal PoISAR observations using polarimetric decomposition techniques
}

Hongtao Shi ${ }^{\text {a, }}$, Lingli Zhao ${ }^{\text {b*, }}$, Jie Yang ${ }^{\text {a }}$, Juan M. Lopez-Sanchez ${ }^{c}$, Jinqi Zhao ${ }^{\text {a }}$, Weidong Sun a , Lei Shi ${ }^{\text {a }}$, Pingxiang $\mathrm{Li}^{\text {a }}$

\footnotetext{
${ }^{\text {a }}$ State Key Laboratory of Information Engineering in Surveying, Mapping, and Remote Sensing, Wuhan University, Wuhan, 430079, China; sht9010@whu.edu.cn; yangj@whu.edu.cn; masurq@whu.edu.cn; widensun2012@whu.edu.cn; shi.lei@whu.edu.cn; pxli@whu.edu.cn

${ }^{\mathrm{b}}$ School of Remote Sensing and Information Engineering, Wuhan University, Wuhan 430079, China

c Institute of Computing Research, University of Alicante, P.O. Box 99, Alicante E-03080, Spain; juanma.lopez@ua.es

*Correspondence: zhaolingli@whu.edu.cn
}

ABSTRACT: Surface soil moisture (SM) retrieval over agricultural areas from polarimetric synthetic aperture radar (PolSAR) has long been restricted by vegetation attenuation, simplified polarimetric scattering modelling, and limited SAR measurements. This study proposes a modified polarimetric decomposition framework to retrieve SM from multi-incidence and multitemporal PolSAR observations. The framework is constructed by combining the X-Bragg model, the extended double Fresnel scattering model and the generalised volume scattering model (GVSM). Compared with traditional decomposition models, the proposed framework considers the depolarisation of dihedral scattering and the diverse vegetation contribution. Under the assumption that SM is invariant for the PolSAR observations at two different incidence angles and that vegetation scattering does not change between two consecutive measurements, analytical parameter solutions, including the dielectric constant of soil and crop stem, can be obtained by solving multivariable nonlinear equations. The proposed framework is applied to the time series of L-band uninhabited aerial vehicle synthetic aperture radar data acquired during the Soil Moisture Active Passive Validation Experiment in 2012. In this study, we assess retrieval performance by comparing the inversion results with in-situ measurements over bean, canola, corn, soybean, wheat and winter wheat areas and comparing the different performance of SM retrieval between the GVSM and Yamaguchi volume scattering models. Given that SM estimation is inherently influenced by crop phenology and empirical parameters which are introduced in the scattering models, we also investigate the influence of surface depolarisation angle and co-pol phase difference on SM estimation. Results show that the proposed retrieval framework 
provides an inversion accuracy of RMSE $<6.0 \%$ and a correlation of $R \geq 0.6$ with an inversion rate larger than $90 \%$. Over wheat and winter wheat fields, a correlation of 0.8 between SM estimates and measurements is observed when the surface scattering is dominant. Specifically, stem permittivity, which is retrieved synchronously with SM also shows a linear relationship with crop biomass and plant water content over bean, corn, soybean and wheat fields. We also find that a priori knowledge of surface depolarisation angle, co-pol phase difference and adaptive volume scattering could help to improve the performance of the proposed SM retrieval framework. However, the GVSM model is still not fully adaptive because the co-pol power ratio of volume scattering is potentially influenced by ground scattering.

Keywords: Synthetic aperture radar; Polarimetric decomposition; Multi-incidence; Multitemporal; Soil moisture.

\section{Introduction}

Soil moisture (SM) is a key parameter in hydrological, meteorological, and climatological modelling (Hajnsek et al., 2003; Huang et al., 2016a; Iodice et al., 2013; Jagdhuber et al., 2013; Karthikeyan et al., 2017a; Wang et al., 2017) as it influences the exchange of water, carbon and heat flux between the atmosphere and the land surface (Karthikeyan et al., 2017b; Sánchez-Ruiz et al., 2014; Zhuo et al., 2016). It also provides essential information for drought forecasting, flood prediction and crop management (Di Martino et al., 2016; El Hajj et al., 2016). Over the past five decades, several techniques have been developed to measure SM, which can be broadly classified into two major groups (Peng et al., 2017): (1) in-situ observations with ground instruments and (2) remote sensing techniques. In-situ methods are characterised by easy installation and the capability of measuring SM at different depths with high accuracy. However, given the large spatial heterogeneity, these observations provide only sparse information and cannot represent the spatial distribution of SM over large areas (Crow et al., 2012; Njoku et al., 2003; Zreda et al., 2012).

Fortunately, active and passive microwave remote sensing techniques can measure SM from the regional to the global scale (De Zan et al., 2014; Fang et al., 2019; Paloscia et al., 2013; Peng et al., 2017; Shi et al., 2019; Wang et al., 2019). Passive remote sensing systems, such as the Advanced Microwave Scanning Radiometer for the Earth Observing System (Njoku et al., 2003), the Soil Moisture and Ocean Salinity satellite (Kerr et al., 2010) and the Soil Moisture Active Passive (SMAP) satellite (Chen et al., 2018), measure the emission (normally the brightness temperature) of the land surface, allowing researchers to find the 
relationship between the observations and soil water content. Although passive techniques can provide a high accuracy (less than $4.0 \%$ ) of SM retrieval, the products have a rather coarse spatial resolution in the order of tens of kilometres (Chen et al., 2018) limited by the antenna dimension of the sensors. Specifically, Copernicus Project Sentinel-1A/1B synthetic aperture radar (SAR) data has proven to be an applicable dataset to disaggregate SMAP radiometer measurements and provide the SM product at much finer resolutions (1 and $3 \mathrm{~km}$ ) at a global extent (Das et al., 2019; Mousa et al., 2020). Furthermore, the proposal of the observation-driven approach to active-passive microwave covariation estimation based on a forward model opens up a new line in combining data from multiple active and passive platforms in terms of high-resolution SM mapping (Jagdhuber et al., 2019).

Active microwave remote sensing techniques, especially SAR, can provide data with a high spatial resolution $(\sim 10-20 \mathrm{~m})$. Polarimetric SAR (PolSAR) is one such technique that can obtain multiple physical properties of the scattering layer. Moreover, polarimetric decomposition (PD) algorithms (Cloude and Pottier, 1997), including eigenvalue- and model-based PD models (Cloude, 2010), have shown that fully polarimetric SAR backscatter signals can be decomposed into three scattering components (i.e. surface, dihedral and volume) (Singh and Yamaguchi, 2018; Xie et al., 2016; Yamaguchi et al., 2005; Cui et al., 2014). However, decomposition methods are commonly used for qualitative analyses, such as image interpretation and classification (Srikanth et al., 2016; Whelen and Siqueira, 2017; Zhao et al., 2014), but not for quantitative applications (van Zyl et al., 2011). For instance, in cases where the ratios of the scattering components from the different decompositions are similar, the classification results may be the same, but the SM estimates would differ greatly because of the inaccurate ground scattering power. Therefore, appropriate decomposition approaches need to be developed to extract the ground scattering power and surface parameters, e.g. SM and soil roughness, accurately (Hajnsek et al., 2003; Jackson et al., 1997; Lunt et al., 2005; Mattia et al., 1997; Schuler et al., 2002; Ulaby et al., 1996). Moreover, SM information can now be estimated by active sensors at different frequencies, incidences and polarisations (Sekertekin et al., 2018; Zhu et al., 2019a, 2019b), with the development of SAR technology.

For SM inversion, Hajnsek et al. (2003) introduced the depolarisation effect into the surface scattering component and proposed the X-Bragg model, which has shown good performances over a wide range of natural surfaces. SM under agricultural vegetation was also investigated by Hajnsek et al. (2009), who 
incorporated the X-Bragg model, the dihedral scattering model and the modified volume scattering model. Jagdhuber et al. (2013) combined the above decomposition model with multi-angular PolSAR data to estimate SM, and their results indicated a remarkable improvement of the inversion rate and a small rootmean-square error (RMSE). Huang et al. (2016a) developed an inversion algorithm which integrates the calibrated integral equation model and the self-adaptive volume scattering model. Recognising that this method disregards the attenuation effect of microwave propagation, Wang et al. (2018) proposed a new SM retrieval algorithm which combines a two-component (surface and volume) PD method and the "Oh 1992" soil scattering model (Oh et al., 1992). Given that the dihedral scattering component is neglected in moderate stalk-dominated vegetation areas, e.g. sugarcane, corn and wheat, the SM inversion rate and accuracy becomes limited when dihedral scattering is considerable. In addition, the assumptions of the surface and dihedral scattering models and the empirical parameters in the model-based PD model intrinsically lead to poor performance in SM estimation over vegetated areas (Antropov et al., 2011; Arii et al., 2010; Freeman and Durden, 1998; Singh and Yamaguchi, 2018; van Zyl et al., 2011; Yamaguchi et al., 2005). To improve the shortcomings of the conventional model-based decompositions, an iterative, generalised, hybrid decomposition algorithm which combines model- and eigenvalue-based techniques was proposed and verified to have a stable performance over different vegetation types (Jagdhuber et al., 2015).

The deficiencies of SM estimation using the model-based PD method can be summarised as two points: (1) the limited PolSAR measurements, simplified scattering models and empirical parameters $(\alpha$ and $\beta)$ which are fixed in accordance with the dominant scattering mechanism (Yamaguchi et al., 2005) in decompositions may lead to inaccurate inversion, and (2) the incorporation of different complex scattering models increases the number of unknown parameters, making SM inversion an ill-posed problem. In this study, we specifically address these problems by (1) introducing a new framework which uses multi-incidence and multitemporal PolSAR data to increase the number of observation parameters and (2) incorporating refined scattering models into the PD model, in which surface and dihedral scattering depolarisation are considered (Jagdhuber, 2012, 2016). Lastly, the derived soil dielectric constant is converted into volumetric soil moisture (mv\%) by utilising the commonly used empirical polynomial of Topp et al. (1980).

The rest of the paper is organised as follows: Section 2 provides the parameterised model-based decomposition model and the framework of the multi-incidence only (MI), the multitemporal only (MT), and 
the multi-incidence and multitemporal (MIMT) PolSAR configuration for SM retrieval. Section 3 describes the selected agricultural study area, the time series of L-band uninhabited aerial vehicle synthetic aperture radar (UAVSAR) PolSAR data and in-situ measurements from Winnipeg. The experimental results of the degree information of multiple PolSAR observations, SM inversion, crop conditions, and the stem dielectric constant estimations are shown in Section 4. Discussions and conclusions are presented in Sections 5 and 6, respectively.

\section{Methodology}

All model-based PD techniques provide a framework to separate the vegetation and ground surface backscattering components from PolSAR measurements (Di Martino et al., 2016; Huang et al., 2016b). Therefore, SM information, which depends on soil dielectric properties, can be extracted from ground-related scattering components (i.e. surface and dihedral scattering). Freeman and Durden (1998) proposed a threecomponent decomposition model, and many other decomposition methods have since been developed on this basis under the reflection symmetry assumption (Arii et al., 2011; Cui et al., 2012; Chen et al., 2014; Singh and Yamaguchi, 2018; An et al., 2010; Yamaguchi et al., 2005). The models of the three different canonical scattering components are introduced as below.

\subsection{Polarimetric decomposition model}

\subsubsection{X-Bragg surface scattering model}

The original surface scattering model used in the model-based decomposition method is the Bragg model, which is derived from Maxwell equations and is suitable for relatively smooth surfaces $(k s<0.3$, where $k$ is the wavenumber, and $s$ is the root-mean-square height) (Jagdhuber et al., 2013). Given that the Bragg scattering model is only valid for a limited surface roughness condition, Hajnsek et al. (2003) developed the X-Bragg scattering model, which considers a wider surface roughness validity range $(k s<$ 1.0). The polarimetric coherency scattering matrix $T_{S_{-} X-B r a g g}$ can be given by (Hajnsek et al. 2003)

$$
\begin{gathered}
{\left[T_{S_{-} X-B_{\mathrm{mag}}}\right]=f_{S}\left[\begin{array}{ccc}
1 & \beta^{*} \operatorname{sinc}(2 \delta) & 0 \\
\beta \operatorname{sinc}(2 \delta) & \frac{1}{2}|\beta|^{2}(1+\operatorname{sinc}(4 \delta)) & 0 \\
0 & 0 & \frac{1}{2}|\beta|^{2}(1-\operatorname{sinc}(4 \delta))
\end{array}\right],} \\
\beta=\frac{R_{H H}-R_{V V}}{R_{H}+R_{V V}},-1<\beta<0,
\end{gathered}
$$




$$
R_{H H}=\frac{\cos \theta-\sqrt{\varepsilon-(\sin \theta)^{2}}}{\cos \theta+\sqrt{\varepsilon-(\sin \theta)^{2}}}, R_{V V}=\frac{(\varepsilon-1)\left((\sin \theta)^{2}-\varepsilon\left(1+(\sin \theta)^{2}\right)\right)}{\left(\varepsilon \cos \theta+\sqrt{\varepsilon-(\sin \theta)^{2}}\right)^{2}},
$$

where $f_{s}$ is the surface scattering amplitude, and $\beta$ is the scattering coefficient related to the incidence angle $\theta$ and relative dielectric constant $\varepsilon . R_{H H}$ and $R_{V V}$ are the Bragg coefficients in the horizontal and vertical polarisations, respectively, which can be depicted as Eq. (3). $\delta$ is the orientation angle induced by the surface slope, which governs the surface scattering depolarisation effect and the cross-polarisation power (Wang et al., 2017).

\subsubsection{Extended dihedral scattering model}

The dihedral scattering component comprises the double bounce scattering between the ground surface and the volume scattering elements that return the reflected signal back to the radar antenna (Zebker, 2010). It is usually assumed to be dominated by a simple dihedral reflection. Therefore, the dihedral scattering coherency matrix $T_{D_{-} \text {Fresnel }}$ of the soil-trunk reflection (in vegetated areas) and ground-wall reflection (in a building area) can be modelled as (Jagdhuber, 2016)

$$
\left[T_{D_{-} \text {Fresnel }}\right]=f_{d}\left[\begin{array}{ccc}
|\alpha|^{2} & \alpha & 0 \\
\alpha^{*} & 1 & 0 \\
0 & 0 & 0
\end{array}\right], \alpha=\frac{R_{H s} R_{H t}-R_{V s} R_{V t} e^{i \Delta \varphi}}{R_{H s} R_{H t}+R_{V s} R_{V t} e^{i \Delta \varphi}}
$$

where $f_{d}$ is the dihedral scattering amplitude; $\alpha$ is the scattering coefficient; $\Delta \varphi$ accounts for the phase difference between the horizontal and vertical polarisations; $R_{H s}$ and $R_{V s}$ are the horizontal and vertical Fresnel scattering coefficients of the soil, respectively; $R_{H t}$ and $R_{V t}$ denotes the horizontal and vertical Fresnel scattering coefficients of the trunk, respectively. The Fresnel scattering coefficients are given by

$$
\left\{\begin{array}{c}
R_{H s}=\frac{\cos \theta-\sqrt{\varepsilon_{\text {soil }}-(\sin \theta)^{2}}}{\cos \theta+\sqrt{\varepsilon_{\text {soil }}-(\sin \theta)^{2}}}, R_{V s}=\frac{\varepsilon_{\text {soil }} \cos \theta-\sqrt{\varepsilon_{\text {soil }}-(\sin \theta)^{2}}}{\varepsilon_{\text {soil }} \cos \theta+\sqrt{\varepsilon_{\text {soil }}-(\sin \theta)^{2}}} \\
R_{H t}=\frac{\cos \left(\frac{\pi}{2}-\theta\right)-\sqrt{\varepsilon_{t / s}-(\cos \theta)^{2}}}{\cos \left(\frac{\pi}{2}-\theta\right)+\sqrt{\varepsilon_{t / s}-(\cos \theta)^{2}}}, R_{V t}=\frac{\varepsilon_{t / s}\left(\frac{\pi}{2}-\theta\right)-\sqrt{\varepsilon_{t / s}-(\cos \theta)^{2}}}{\varepsilon_{t / s}\left(\frac{\pi}{2}-\theta\right)+\sqrt{\varepsilon_{t / s}-(\cos \theta)^{2}}}
\end{array}\right.
$$

where $\varepsilon_{\text {soil }}$ and $\varepsilon_{t / s}$ are the dielectric constants of soil and trunks or stems which are vertically oriented with respect to ground, respectively. This double Fresnel scattering model has been widely used in modelbased decomposition. However, as in the Bragg model, it also neglects the dihedral scattering depolarisation effect induced by soil roughness. To deal with this problem, Jagdhuber (2016) proposed an extended Fresnel scattering model for the modelling of depolarising double-bounce scattering. Similar to the X-Bragg model, the polarimetric coherency scattering matrix $T_{D \_X-F r e s n e l}$ for the extended double Fresnel scattering model is given by 


$$
\left[T_{D_{-} X-\text { Fresnel }}\right]=f_{d}\left[\begin{array}{ccc}
\frac{T_{X D 11}}{T_{X D 22}} & \frac{T_{X D 12}}{T_{X D 22}} & 0 \\
\frac{T_{X D 21}}{T_{X D 22}} & 1 & 0 \\
0 & 0 & \frac{T_{X D 33}}{T_{X D 22}}
\end{array}\right]
$$

where $f_{d}$ is the dihedral scattering amplitude. The Bragg $\left(T_{S_{-} B \text { ragg }}\right)$ and double Fresnel scattering models $\left(T_{D_{-} \text {Fresnel }}\right)$ are rank-1 matrix, whereas the X-Bragg $\left(T_{S_{-} X-B \text { ragg }}\right)$ and extended double Fresnel scattering models $\left(T_{D_{-} X-\text { Fresnel }}\right)$ are rank-3. Details of $T_{X D 11}, T_{X D 12}, T_{X D 22}$ and $T_{X D 33}$ in Eq. (6) could be found in (Jagdhuber, 2016).

\subsubsection{Generalised volume scattering model}

The volume scattering component in model-based PD is usually modelled as a cloud of randomly oriented spherical, oblate spherical, or prolate spherical particles (Freeman and Durden, 1998; Zebker, 2010), e.g. the volume scattering model of Yamaguchi et al. (2005). The GVSM model proposed by Antropov et al. (2011) exploits the co-polarimetric ratio $(\gamma)$ and the correlation coefficient (fixed at $\rho=1 / 3$ ) from data to account for either dominant horizontal or vertical orientation of vegetation elements in the canopy. As discussed in the literature (Antropov et al., 2011), Yamaguchi et al. (2005) provided three volume scattering models, i.e. horizontally, vertically and randomly aligned cloud dipoles, to describe the canopy scattering in a step-wise form on the basis of the co-pol power ratio. The dependence of a canopy model on PolSAR observations is preferred to have a continuous form. The GVSM is general enough to allow a broad range of canopies to be modelled, and it complies with several earlier proposed volume scattering mechanism models (Mandal et al., 2020; Ratha et al., 2019). Hence, it is embedded into the newly developed PD framework to make it applicable for SM estimation in different vegetation types. As shown in Eq. (7), the coherency matrix of the GVSM is given as

$$
\left[T_{V}\right]^{\text {Orien }}=\frac{f_{v}}{3+3 \gamma-\frac{2 \sqrt{\gamma}}{3}}\left[\begin{array}{ccc}
\gamma+\frac{2 \sqrt{\gamma}}{3}+1 & \gamma-1 & 0 \\
\gamma-1 & \gamma-\frac{2 \sqrt{\gamma}}{3}+1 & 0 \\
0 & 0 & \gamma-\frac{2 \sqrt{\gamma}}{3}+1
\end{array}\right]
$$

where the co-polarised ratio $\gamma$ is defined as $\left\langle\left|S_{H H}\right|^{2}\right\rangle /\left\langle\left|S_{V V}\right|^{2}\right\rangle$. The advantage of using GVSM in SM estimation algorithms could be found in Section 4.2, which shows the comparisons of SM estimates between the volume scattering model of Yamaguchi et al. and GVSM.

2.2. SM inversion based on multi-incidence and multitemporal decomposition framework 
Considering the depolarisation effects of the surface and dihedral scattering components, we firstly incorporate the X-Bragg model, the extended dihedral scattering model and the generalised volume scattering model in the decomposition framework, leading us to (8):

$$
[T]=\left[\begin{array}{ccc}
T_{11} & T_{12} & 0 \\
T_{12}^{*} & T_{22} & 0 \\
0 & 0 & T_{33}
\end{array}\right]=\left[T_{S_{-} X-B_{\mathrm{mag}}}\right]+\left[T_{D_{-} X-\text { Fresnel }}\right]+\left[T_{V}\right]^{\text {Orien }},
$$

As shown in (1)-(7), seven parameters, i.e. $f_{s}, f_{d}, f_{v}, \varepsilon_{\text {soil }}, \varepsilon_{t / s}, \delta$ and $\Delta \varphi$ in (8), exist, whereas $\delta$ and $\Delta \varphi$ can be determined empirically in accordance with Hajnsek et al. (2009). In this case, it leaves only four observations, i.e. $T_{11}, T_{12}, T_{22}$ and $T_{33}$, in five unknowns, making the proposed inversion method an illposed problem. In addition, the conventional model-based decomposition method usually needs to determine the dominant scattering mechanism (surface or dihedral) and set empirical parameters ( $\alpha$ or $\beta$ ) when solving the unknown parameters due to the limited observations. The ill-posed problem and empirical parameters inevitably reduce the accuracy of the decomposition results. One way to solve the empirical setting of $\alpha$ and $\beta$ is through an eigenvalue-based decomposition method (Jagdhuber et al., 2015). Alternatively, the multimode imaging modalities of the PolSAR technique (Fig. 1) can provide the possibility of increasing the observation information (i.e. number of equations) and solving the unknown parameters. Here, we propose to use the repeated observations at two different incidence angles and/or on two consecutive passes, i.e. multiincidence and/or multitemporal PolSAR data, to calculate the unknown parameters, including the dielectric constant of soil $\left(\varepsilon_{\text {soil }}\right)$ and stem $\left(\varepsilon_{t / s}\right)$, and the scattering power coefficient of surface $\left(f_{s}\right)$, dihedral $\left(f_{d}\right)$, and volume $\left(f_{v}\right)$ scattering components.

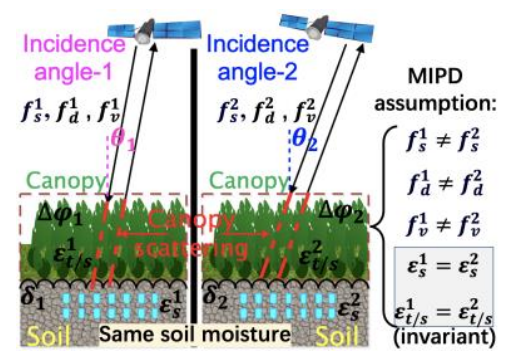

(a)

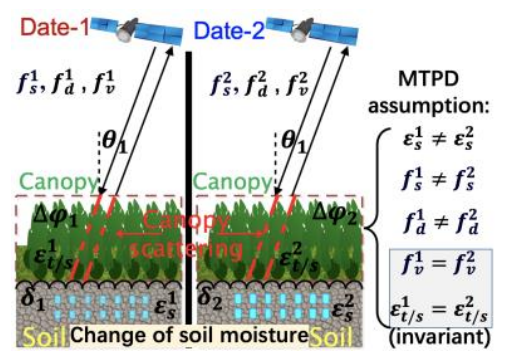

(b)

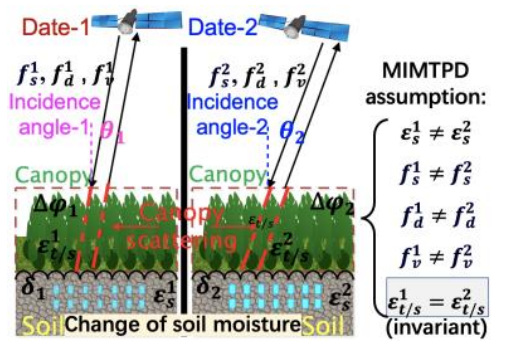

(c)

Fig. 1. Multiple observation PolSAR imaging modalities for SM inversion. (a) Multi-incidence and angular observation mode. (b) Multitemporal observation mode. (c) Multi-incidence and multitemporal observation mode. $\varepsilon_{s}^{1}$ and $\varepsilon_{s}^{2}$ are the soil dielectric constants at different surface depths; $\varepsilon_{t / s}^{1}$ and $\varepsilon_{t / s}^{2}$ are the trunk or stem dielectric constants; $\delta_{1}$ and $\delta_{2}$ are the depolariaation angles; $\Delta \varphi_{1}$ and $\Delta \varphi_{2}$ are the phase difference between horizontal and vertical polarisations of the vegetation canopy; $\theta_{1}$ and $\theta_{2}$ denote the different incidence angles at the same looking direction; $f_{s}^{1}, f_{d}^{1}, f_{v}^{1}, f_{s}^{2}, f_{d}^{2}$ and $f_{v}^{2}$ are the coefficients of the coherency matrix of the surface, dihedral, and volume scattering components from different observations.

Assuming quad-pol SAR data from two different configurations are available, the simultaneous equations of the coherency matrix have the general form shown in Eq. (9), which is termed as the multiple 
observation PD model in this study.

$$
\begin{aligned}
& {[T]^{O 1}=\left[T_{S_{-} X-\mathrm{Bragg}}\right]^{1}+\left[T_{D_{-} X-\text { Fresnel }}\right]^{1}+\left[T_{G V}\right]^{\text {Orien-1 }}} \\
& {[T]^{O 2}=\left[T_{S_{-} X-\mathrm{Bragg}}\right]^{2}+\left[T_{D_{-} X-\text { Fresnel }}\right]^{2}+\left[T_{G V}\right]^{\text {Orien-2 }}}
\end{aligned}
$$

where $[T]^{01}$ and $[T]^{02}$ are the coherency matrices of the two different observations under reflection symmetry assumption $\left(T_{13}=T_{23}=0\right)$. The number of observation equations is then increased from four to eight (i.e. $T_{11}^{O 1}, T_{12}^{O 1}, T_{22}^{O 1}, T_{33}^{O 1}, T_{11}^{O 2}, T_{12}^{O 2}, T_{22}^{O 2}$ and $T_{33}^{O 2}$ ). PD and $\mathrm{SM}$ inversion are eventually turned into a problem of solving multivariable nonlinear equations, as shown in Eq. (10).

$$
\left\{\begin{array}{c}
T_{11}^{O 1}=f_{s}{ }^{1}+f_{d}{ }^{1} \cdot\left(T_{X D 11_{1}} / T_{X D 22_{1} 1}\right)+f_{v}{ }^{1} \cdot\left[\left(\gamma_{1}+2 \sqrt{\gamma_{1}} / 3+1\right) /\left(3+3 \gamma_{1}-2 \sqrt{\gamma_{1}} / 3\right)\right] \\
T_{12}^{O 1}=f_{s}{ }^{1} \cdot \beta^{*} \operatorname{sinc}(2 \delta)+f_{d}{ }^{1} \cdot\left(T_{X D 12_{1} 1} / T_{X D 22_{1} 1}\right)+f_{v}{ }^{1} \cdot\left[\left(\gamma_{1}-1\right) /\left(3+3 \gamma_{1}-2 \sqrt{\gamma_{1}} / 3\right)\right] \\
T_{22}^{O 1}=f_{s}{ }^{1}+0.5 f_{d}{ }^{1} \cdot \beta^{2} \cdot\left[1+\operatorname{sinc}\left(4 \delta^{1}\right)\right]+f_{v}{ }^{1} \cdot\left[\left(\gamma_{1}-2 \sqrt{\gamma_{1}} / 3+1\right) /\left(3+3 \gamma_{1}-2 \sqrt{\gamma_{1}} / 3\right)\right] \\
T_{33}^{O 1}=f_{s}{ }^{1}+0.5 f_{d}{ }^{1} \cdot \beta^{2} \cdot\left[1-\operatorname{sinc}\left(4 \delta^{1}\right)\right]+f_{v}{ }^{1} \cdot\left[\left(\gamma_{1}-2 \sqrt{\gamma_{1}} / 3+1\right) /\left(3+3 \gamma_{1}-2 \sqrt{\gamma_{1}} / 3\right)\right] \\
T_{11}^{O 2}=f_{s}{ }^{2}+f_{d}{ }^{2} \cdot\left(T_{X D 11_{2}} / T_{X D 22_{2} 2}\right)+f_{v}{ }^{2} \cdot\left[\left(\gamma_{2}+2 \sqrt{\gamma_{2}} / 3+-1\right) /\left(3+3 \gamma_{2}-2 \sqrt{\gamma_{2}} / 3\right)\right] \\
T_{12}^{O 2}=f_{s}{ }^{2} \cdot \beta^{*} \operatorname{sinc}(2 \delta)+f_{d}{ }^{2} \cdot\left(T_{X D 12_{2} 2} / T_{X D 22_{2} 2}\right)+f_{v}{ }^{2} \cdot\left[\left(\gamma_{2}-1\right) /\left(3+3 \gamma_{2}-2 \sqrt{\gamma_{2}} / 3\right)\right] \\
T_{22}^{O 2}=f_{s}{ }^{2}+0.5 f_{d}{ }^{2} \cdot \beta^{2} \cdot\left[1+\operatorname{sinc}\left(4 \delta^{2}\right)\right]+f_{v}{ }^{2} \cdot\left[\left(\gamma_{2}-2 \sqrt{\gamma_{2}} / 3+1\right) /\left(3+3 \gamma_{2}-2 \sqrt{\gamma_{2}} / 3\right)\right] \\
T_{33}^{O 2}=f_{s}{ }^{2}+0.5 f_{d}{ }^{2} \cdot \beta^{2} \cdot\left[1-\operatorname{sinc}\left(4 \delta^{2}\right)\right]+f_{v}{ }^{2} \cdot\left[\left(\gamma_{2}-2 \sqrt{\gamma_{2}} / 3+1\right) /\left(3+3 \gamma_{2}-2 \sqrt{\gamma_{2}} / 3\right)\right]
\end{array}\right.
$$

For the two different quad-pol SAR images, the unknown parameters, i.e. $f_{s}, f_{d}$ and $f_{v}$, may change with incidence angle and time. In addition, the local incidence angles of the same pixel in two different observations (i.e. $\theta^{1}(i, j)$ and $\theta^{2}(i, j)$, where $(i, j)$ is the pixel location) can be obtained from the SAR system parameters. Although the multi-observation data could be used for PD, it needs to set some proper assumptions for the different imaging modalities (Fig. 1) as follows: (1) For the MI observation mode (i.e. Fig. 1a), assuming that the soil dielectric constant $\left(\varepsilon_{\text {soil }}\right)$, the trunk or stem dielectric constant $\left(\varepsilon_{t / s}\right)$ and the phase difference $(\Delta \varphi)$ are invariant is reasonable; (2) for the MT observations (i.e. Fig. 1b), in a short time interval, the scattering power of volume $\left(f_{v}\right)$ scattering components, the phase difference $(\Delta \varphi)$, the trunk or stem dielectric constant $\left(\varepsilon_{t / s}\right)$ and the depolarisation angle $(\delta)$ can be assumed to be invariant, whereas the scattering power of the surface $\left(f_{s}\right)$ and dihedral $\left(f_{d}\right)$ and soil dielectric constant $\left(\varepsilon_{\text {soil }}\right)$ values are different; (3) for the MIMT observations (i.e. Fig. 1c), $\varepsilon_{t / s}$ is assumed to be unchanged between two consecutive observations at different incidence angles. Under these assumptions, the number of the unknown parameters of Eq. (10) for MIPD and MTPD algorithms increases to 12, whereas that for MIMTPD increases to 13, which is still larger than the number of measurement equations. In other words, Eq. (10) remains undetermined even after introducing the MIMT PolSAR observations. However, a determined system of equation could be made empirically by setting the surface depolarisation angle $(\delta)$ and co-pol phase 
difference $(\Delta \varphi)$.

According to Hajnsek et al. (2003), Jagdhuber et al. (2013), Mattia et al. (1997) and Schuler et al. (2002), the width $(\delta)$ of the angular distribution of the facets introduces depolarisation and a cross-polarised signal. In addition, the polarimetric circular coherence is independent of the surface dielectric constant, and is often taken in preference as the roughness estimator. Therefore, we use the polarimetric circular coherence to estimate the empirical parameter $\delta$ (Mattia et al. 1997)

$$
\delta=\frac{\pi}{2}\left(1-\left|\gamma_{R R L L}\right|\right), \gamma_{R R L L}=\frac{\left\langle S_{R R} S_{L L}^{*}\right\rangle}{\sqrt{\left\langle\left|S_{R R}\right|^{2}\right\rangle\left\langle\left|S_{L L}\right|^{2}\right\rangle}}
$$

where $\gamma_{R R L L}$ is the polarimetric circular coherence. $S_{R R}$ and $S_{L L}$ are the scattering coefficients in the right-circular and the left-circular copolarisations, respectively. As described in Eq. (5) and (6), $\Delta \varphi$ exists only in the extended dihedral scattering model. In this case, the sensitivity of the extended dihedral scattering model regarding $\Delta \varphi$ can be investigated on the basis of a forward model. Jagdhuber (2016) analysed the influence of $\Delta \varphi$ on different element combinations of matrix $T_{D_{-} X-F r e s n e l}$, and concluded that the phase difference $(\Delta \varphi)$ has a low impact on the coherency matrix combination. Hence, the polarimetric phase difference $(\Delta \varphi)$ is neglected and empirically set to zero (Hajnsek et al., 2009). The zero $\Delta \varphi$ assumes a randomly oriented vegetation volume structure and neglects the anisotropies in scattering. However, for the crop which has vertical stalks and a dominating structure, the dihedral scattering could not be modelled correctly. With regard to the uncertainty of $\Delta \varphi$ in the decomposition model, a detailed analysis of the influence of $\Delta \varphi$ on the SM estimation over different crop fields is provided in Section 4.5.1.

\subsection{Nonlinear equation solution and unknown parameter constraints}

In the proposed decomposition model, we empirically set $\delta=\pi *\left(1-\left|\gamma_{R R L L}\right|\right) / 2$ and $\Delta \varphi=0$. The two parameters are thoroughly investigated to understand the impact and further optimise results (Section 4.5). With the empirical values of $\delta$ and $\Delta \varphi$, the number of unknown parameters of Eq. (10) for MIPD and MTPD decreases to eight, which is equal to the number of measurement equations. Therefore, the decomposition model which is based on multiple PolSAR observations is now determined, and the multivariable nonlinear equations have positive definite solutions (i.e. $f_{s}{ }^{1}, f_{d}{ }^{1}, f_{v}{ }^{1}, f_{s}{ }^{2}, f_{d}{ }^{2}, f_{v}{ }^{2}, \varepsilon_{\text {soil }}$ and $\left.\varepsilon_{t / s}\right)$. In this work, the unknown parameters are obtained by solving the following nonlinear leastsquares curve fitting problem: 


$$
\begin{gathered}
\min _{X}\left\|\begin{array}{l}
{[T]^{O 1}-\left[T_{S_{-} X-\text { Bragg }}\right]^{1}-\left[T_{D_{-} X-\text { Fresnel }}\right]^{1}-\left[T_{V}\right]^{\text {Orien-1 }}} \\
{[T]^{O 2}-\left[T_{S_{-} X-\text { Bragg }}\right]^{2}-\left[T_{D_{-} X-\text { Fresnel }}\right]^{2}-\left[T_{V}\right]^{\text {Orien-2 }}}
\end{array}\right\|_{2}^{2}, \\
X=\left[\begin{array}{llllllll}
f_{s}{ }^{1} & f_{d}{ }^{1} & f_{v}{ }^{1} & f_{s}{ }^{2} & f_{d}{ }^{2} & f_{v}{ }^{2} & \varepsilon_{\text {soil }} & \varepsilon_{t / s}
\end{array}\right],
\end{gathered}
$$

with optional lower and upper bounds of scattering power, soil and vegetation stem dielectric constant in the component of vector $X$ (Table 1). For MIMTPD, although the number of unknowns is nine, i.e. $\varepsilon_{t / s}^{1} \neq \varepsilon_{t / s}^{2}$, the unknown parameters can be calculated from the un-determined system by solving Eq. (12). The boundary conditions of $\varepsilon_{\text {soil }}$ and $\varepsilon_{t / s}$ are selected in accordance with the dielectric properties of natural soils (2-35) and media (4-80) (Mavrovic et al., 2018). Then, SM can be obtained indirectly from the derived soil dielectric constant $\varepsilon_{\text {soil }}$, and the calculated power ratio of the surface, dihedral and volume scattering components can be used to analyse the scattering characteristics of the scene. In consideration of the diversity of the vegetation structure, the influence of $\Delta \varphi$ on SM estimation over the selected crop fields are investigated; findings are included in the Results and Discussion sections. The estimated surface depolarisation angle $\delta$ is inevitably influenced by vegetation growth; hence, the SM estimation using the $\delta$ derived from the observation obtained on the least vegetated date $\left(\delta_{\text {least_veg }}\right)$ is also performed and analysed in this work.

\section{Table 1}

Variation bounds and values of the included variables in the decomposition model.

\begin{tabular}{cccc|ccc}
\hline $\begin{array}{c}\text { Unknown } \\
\text { parameter }\end{array}$ & Meaning & $\begin{array}{c}\text { Lower } \\
\text { bound }\end{array}$ & $\begin{array}{c}\text { Upper } \\
\text { bound }\end{array}$ & $\begin{array}{c}\text { Fixed } \\
\text { parameter }\end{array}$ & Meaning & Value \\
\hline$f_{s}{ }^{1}, f_{d}{ }^{1}, f_{v}{ }^{1}$ & $\begin{array}{c}\text { Surface, dihedral, and } \\
\text { volume scattering power } \\
\text { of data 1 and data 2 }\end{array}$ & 0 & $\begin{array}{c}\text { Total power } \\
\text { of data 1. }\end{array}$ & $\delta$ & $\begin{array}{c}\text { Total power } \\
\text { of data } 2 .\end{array}$ & Depolarisation \\
$f_{s}{ }^{2}, f_{d}{ }^{2}, f_{v}{ }^{2}$ & angle. & $\frac{\left(1-\left|\gamma_{R R L L}\right|\right) * \pi}{2}$ \\
$\varepsilon_{\text {soil }}$ & Soil permittivity. & 2 & 35 & $\Delta \varphi$ & Co-pol phase & 0 \\
$\varepsilon_{t / s}$ & Stem permittivity & 4 & 80 & difference & 0 \\
\hline
\end{tabular}




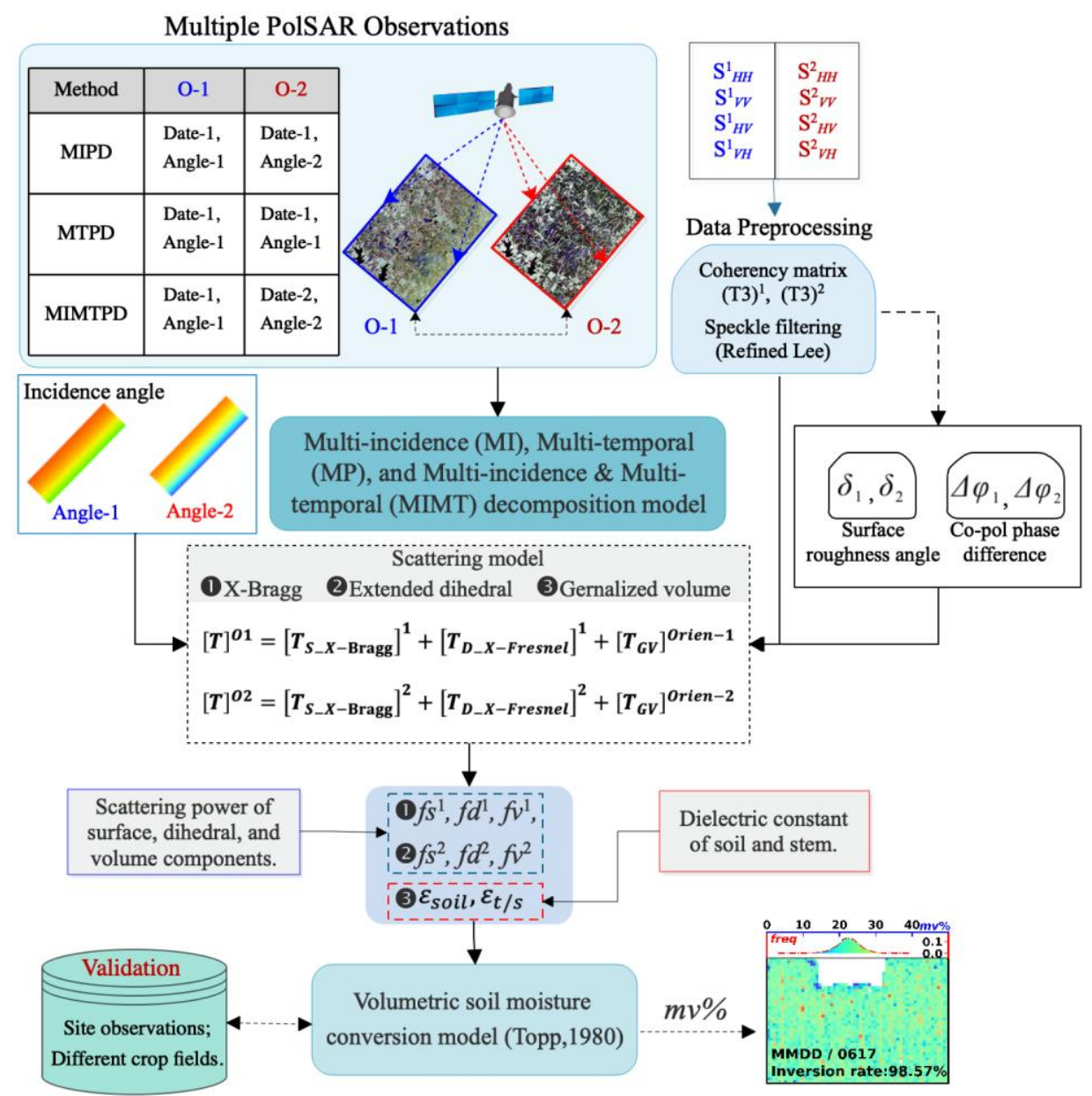

Fig. 2. Workflow of PD based on multiple observations and the SM inversion procedure.

Fig. 2 outlines the workflow of polarimetric decomposition and the SM inversion procedure using multiple quad-pol SAR observations. In this study, the soil dielectric constant is converted to volumetric soil moisture (mv\%) by using a commonly used empirical polynomial of Topp et al. (1980).

\subsection{Degrees of information}

Different observations may not be completely independent. Thus, the degrees of freedom in a set of observations should be investigated and more independent measurements than unknown parameters could retrieved. In this study, the normalised total correlation calculation framework proposed by Konings et al. (2015) is applied to obtain the degrees of information (DoI) of the MI, MT and MIMT PolSAR observations. The DoI of two quad-pol SAR measurements can be calculated as indicated in Appendix A. Specifically, under reflection symmetry assumption, 10 measurements (also termed variables or features) for the PolSAR dataset are obtained at two different incidence angles or dates, i.e. $T_{11}^{O 1}, \mathfrak{R}\left(T_{12}^{O 1}\right), \mathfrak{I}\left(T_{12}^{O 1}\right), T_{22}^{O 1}, T_{33}^{O 1}, T_{11}^{O 2}$, $\Re\left(T_{12}^{O 2}\right), \mathfrak{I}\left(T_{12}^{O 2}\right), T_{22}^{O 2}, T_{33}^{O 2}(\mathrm{~N}=10)$. As introduced in Konings et al. (2015), an appropriate bin size 
determined by Scott's rule and the discretisation of PolSAR continuous measurements are also necessary to evaluate $C_{n}$ and DoI accurately.

\section{Study areas and datasets}

\subsection{UAVSAR multi-incidence and multitemporal observations of Winnipeg, Canada}

UAVSAR is an airborne-based quad-pol L-band radar system equipped on the NASA Gulfstream-III aircraft. The time-series quad-pol UAVSAR data from Winnipeg, Manitoba, Canada were collected as part of the SMAP Validation Experiment 2012 (SMAPVEX12) from June 6 to July 17, 2012. Each acquisition date has four flights, namely, 31603, 31604, 31605 and 31606 (Fig. 3). All the observations looked to the left of the flight direction. Data were collected over a look angle interval from $20^{\circ}$ to $65^{\circ}$, with a spatial resolution of $5.0 \mathrm{~m}$ in the range direction and $7.2 \mathrm{~m}$ in the azimuth direction. The four flight line observations with the same range and azimuth direction have an overlapping area, which allowed us access to four quad-pol images at different incidence angles.

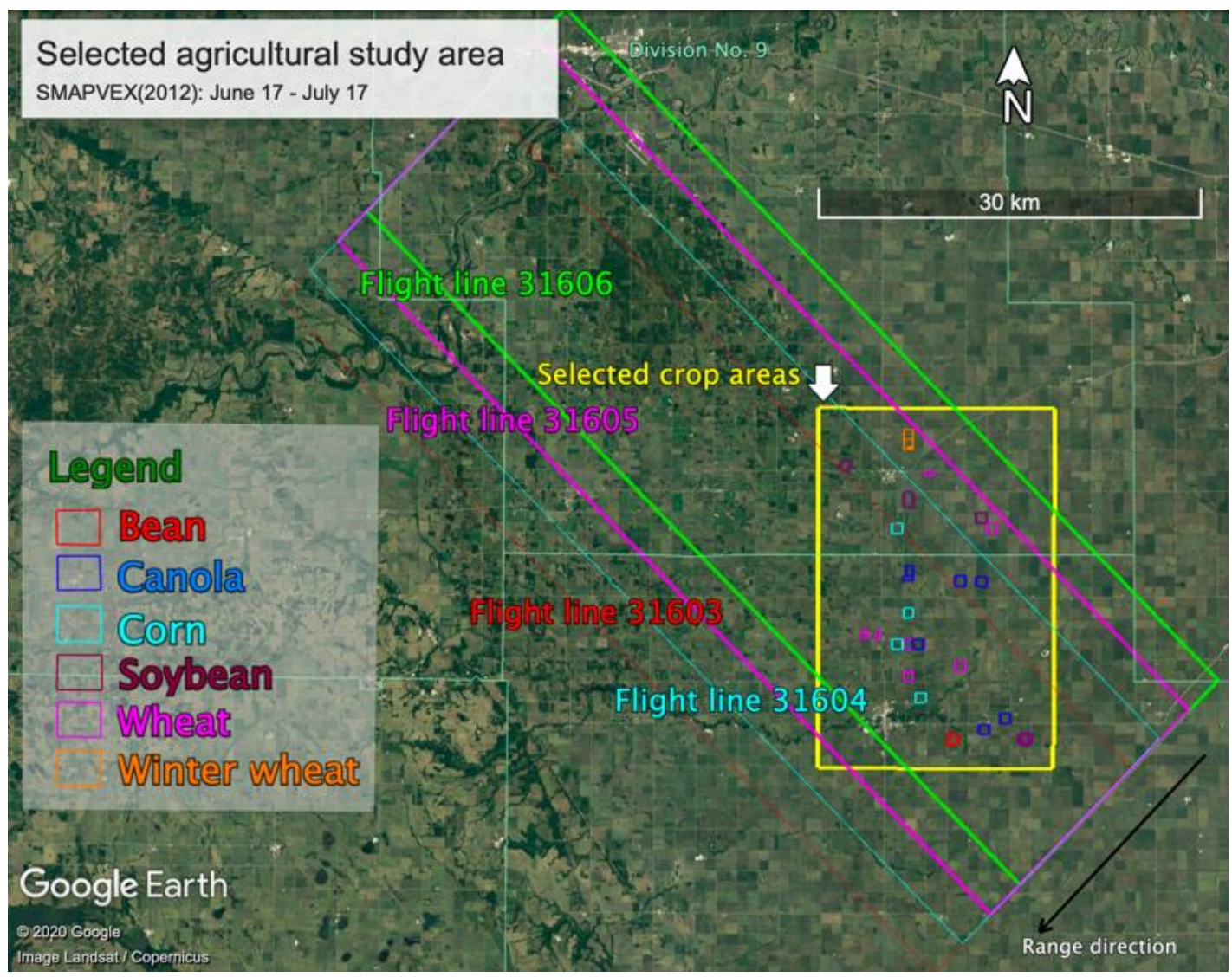

(a) Selected agricultural study area 


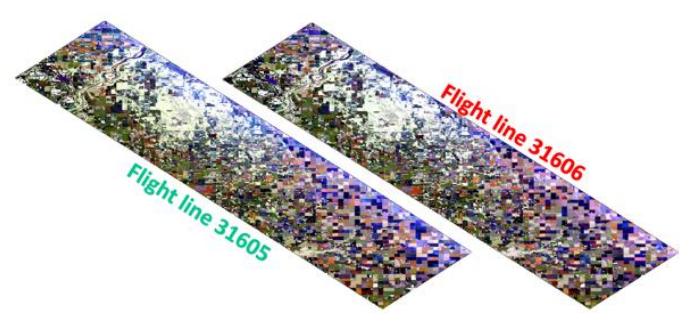

(b) PauliRGB

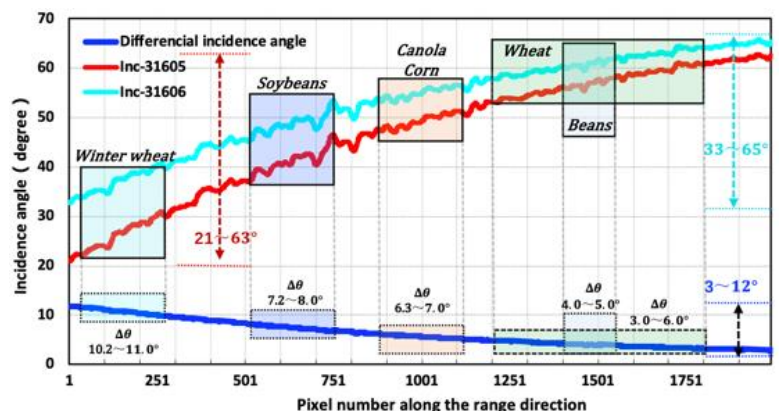

(c) Incidence angle

Fig. 3. (a) UAVSAR flight lines and SAR image coverage areas for the Winnipeg test site. The red, cyan, pink and green rectangles are the four flight lines, namely, 31603, 31604, 31605 and 31606, respectively. (b) PauliRGB of flight line 31605 and 31606. (c) Incidence angle along the range direction of beans, crop, corn, soybeans, wheat and winter wheat crop fields. The red and cyan lines in (c) denote the incidence angle of flight line $31605\left(21^{\circ}-63^{\circ}\right)$ and $31606\left(33^{\circ}-65^{\circ}\right)$, respectively. The blue line presents the incidence angle difference $\left(3^{\circ}-12^{\circ}\right)$ from the near range to far range direction.

For the study area, the time-series observations also enabled us to obtain two PolSAR images between the two dates. Two flight lines (i.e. 31605 and 31606) of the 14 days of UAVSAR observations (28 images) were selected for the SM inversion experiment. Fig. 3 depicts the four UAVSAR flight lines and the selected agricultural study areas in Winnipeg. SAR data were processed by extracting the coherency matrix from the calibrated UAVSAR products, and the $7 \times 7$ refined Lee filter was used to suppress the speckle noise. Then, the dataset was used for three experiments, i.e. SM estimates from MI, MT and MIMT PolSAR observations.

\subsection{Ancillary field survey data and ground measurements}

Given the SMAPVEX12 campaign, field survey data and site measurements were available for us to evaluate the performance of the SM inversion method. The SMAPVEX12 campaign took place in an agricultural region of South Winnipeg, which features a range of crop type. The study area has nine permanent in-situ SM stations operated by Agriculture and Agri-Food Canada (AAFC); 50 temporary stations operated by the United States Department of Agriculture, AAFC and the Manitoba Agriculture, Food and Rural Initiatives. The site observations of volumetric SM and the height, diameter, biomass and area plant water content (PWC) of crop were selected to evaluate the experimental results. In addition, the surface root-mean-square (RMS) height measurements were collected in the look directions of UAVSAR, measured using a digital camera and a $1 \mathrm{~m}$-long pin profilometer consisting of 200 needles spaced with an interval of $5 \mathrm{~mm}$.

\section{Results}

Three experiments, i.e. SM retrieval based on MIPD, MTPD and MIMTPD methods, were 
implemented to investigate the performance of the proposed framework. The retrieval algorithms, assumptions and determination of initial parameters have been described in Sections 2.2 and 2.3. Prior to the three experiments, the DoI information of MI, MT and MIMT PolSAR observations are shown to evaluate the number of unknown variables that can be retrieved. Then, the time series of volumetric SM estimates over bean, canola, corn, soybean, wheat and winter wheat fields are presented together with the in-situ measurements.

4.1. DoI of single and multiple PolSAR observations

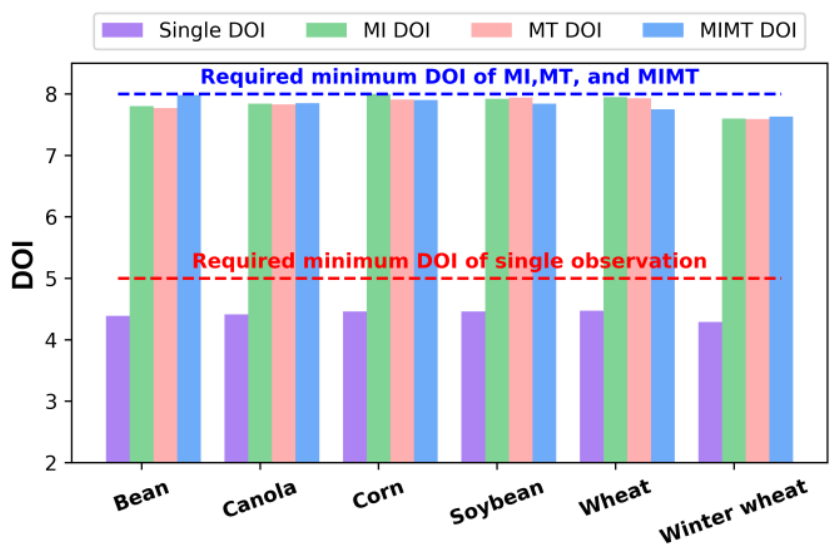

Fig. 4. DoI of the single and multiple PolSAR observations over bean, canola, corn, soybean, wheat and winter wheat agricultural fields.

Generally, the single-acquisition PD model has five unknowns, whereas the multi-incidence or multitemporal PD model has eight unknowns (Section 2.5). Therefore, the required minimum DoI is five and eight for single and multiple PolSAR observations, respectively. In this section, the DoI calculations for MI, MT and MIMT PolSAR SM inversion framework are illustrated in Fig. 4 with the input datasets which are described in Sections 2.2 and 3.1. The calculated DoIs of MI, MT and MIMT PolSAR observations are less than 8 (7.8-8.0). In other words, the number of unknown parameters is larger than the floor of the DoIs. One of the reasons could be the PolSAR noise. Another reason that needs to be considered is the change of the surface parameters (e.g. soil permittivity) and vegetation parameters (e.g. structure and VWC). In this sense, when no change happens between two consecutive PolSAR observations (e.g. multitemporal measurements), the scattering power coefficients of single PolSAR observations (i.e. $f_{s}^{1}, f_{d}^{1}, f_{v}^{1}$ ) are not completely independent of the parameters of another one (i.e. $f_{s}^{2}, f_{d}^{2}, f_{v}^{2}$ ). In other words, the coefficient of the scattering power is more sensitive to the soil or trunk/stem permittivity (SP) instead of the incidence angle. The simulation of $f_{s}$ and $f_{d}$ based on the forward scattering model (Hajnsek et al., 2009) and their sensitivity 
to incidence angle and soil dielectric constant could provide an explanation (Fig. 5).
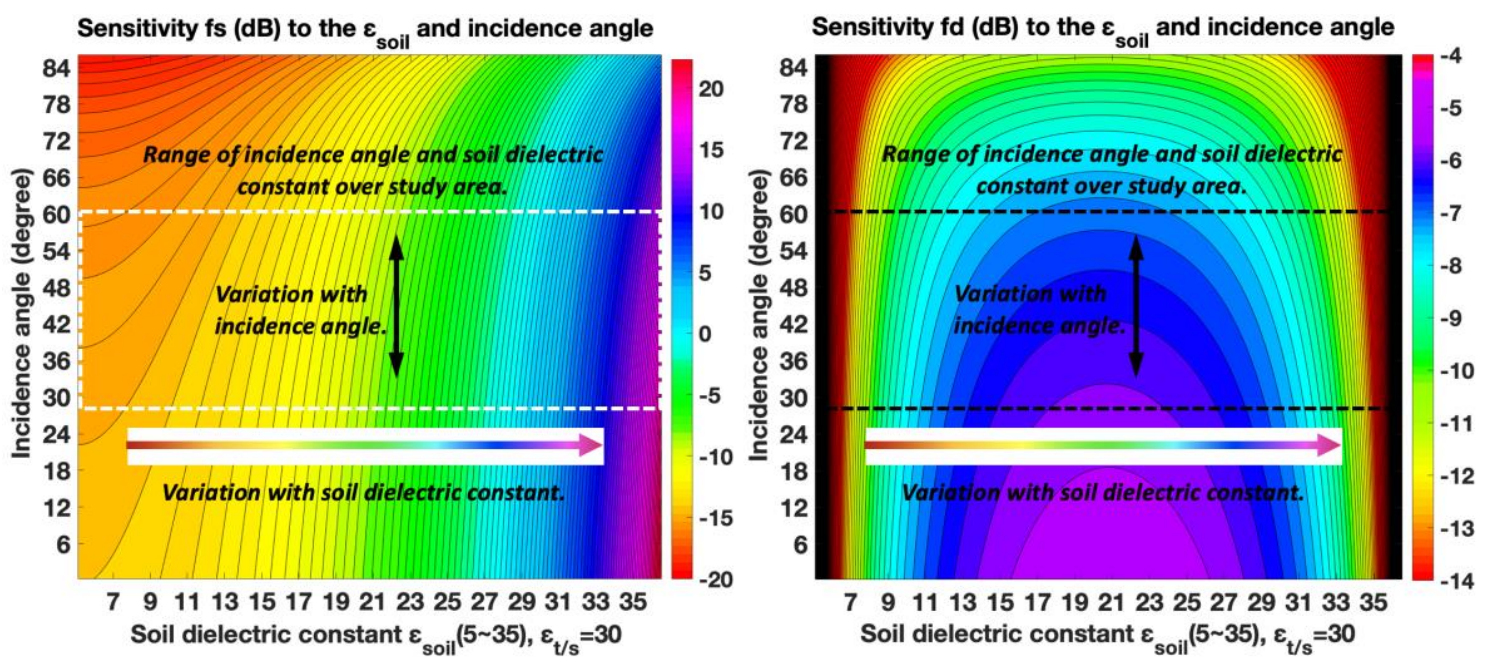

Fig. 5. Sensitivity of $f_{s}$ and $f_{d}$ to incidence angle and soil dielectric constant $\left(\varepsilon_{t / s}=30\right)$.

Fig. 5 indicates that $f_{s}$ is more sensitive to the soil dielectric constant (i.e. ranges from $-15 \mathrm{~dB}$ to $15 \mathrm{~dB}$ ) with a soil dielectric constant ranging from 5 to 35 . In our research area where the difference of the incidence angle ranges from $3^{\circ}$ to $12^{\circ}$, the value of $f_{s}^{1}$ is possibly close to $f_{s}^{2}$. In addition, the simulation of $f_{d}$ from the forward model shows that taking $f_{d}^{1} \approx f_{d}^{2}$ within a certain range of incidence angle and soil permittivity can also be considered in our study. In this case, the number of the unknown parameters in MIPD, MTPD and MIMTPD SM retrieval model could be less than eight. In addition, the unknown parameters are estimated by solving the multivariable nonlinear equations. It could also provide the solutions of an underdetermined system. Therefore, retrieving additional unknown geophysical parameters, e.g. the soil and stem dielectric constant, with additional measurements made at different times, incidence angles or even different electromagnetic frequencies, is possible.

\subsection{Volumetric soil moisture estimation}

The SM retrievals are undertaken using the proposed MIPD, MTPD and MIMTPD models. Two flight lines, i.e. 31605 and 31606 , of the 14 days of L-band UAVSAR quad-pol observations (28 images) were selected for the SM inversion. Considering the different crop types in the study area, we show the evolution of time series SM measurements and estimates over bean, canola, corn, soybean, wheat and winter wheat fields in Fig. 6. 

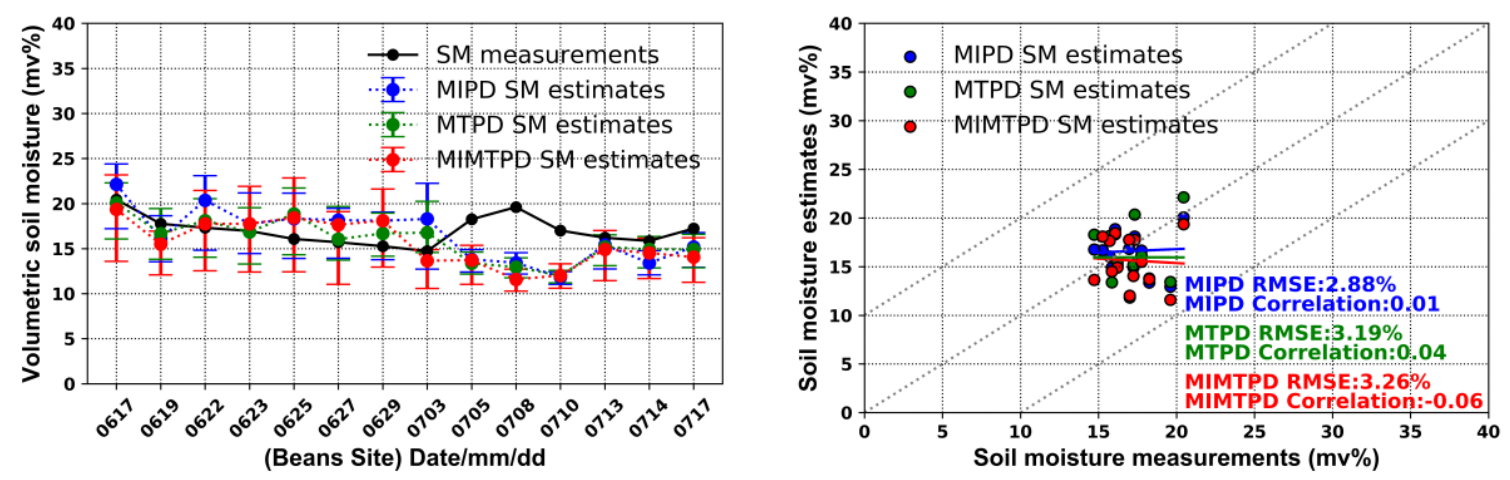

(a) Bean field
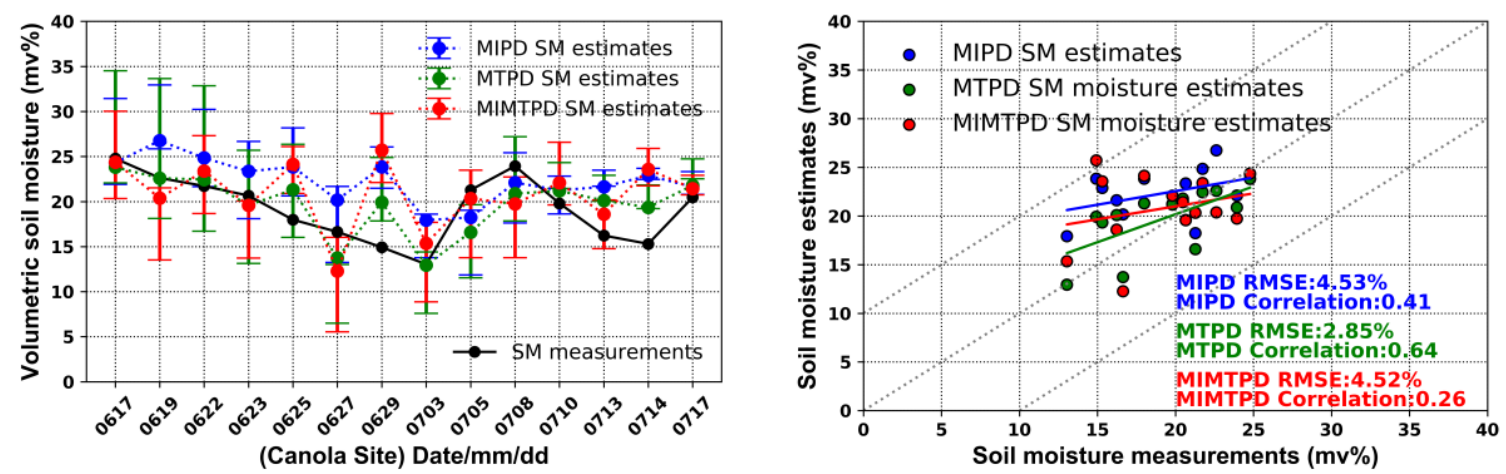

(b) Canola field
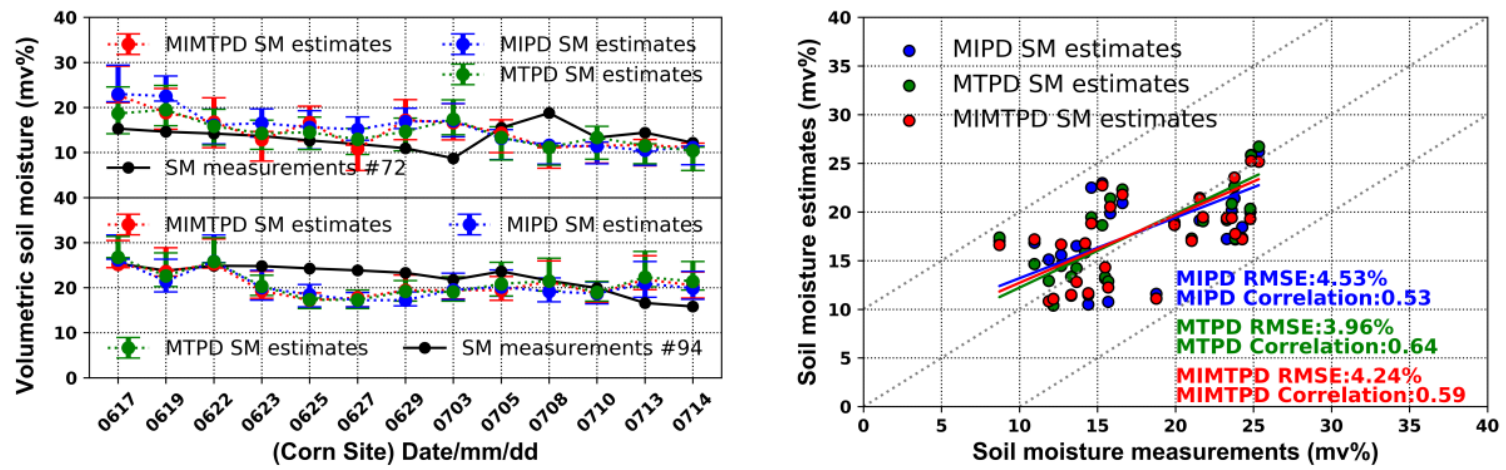

(c) Corn field
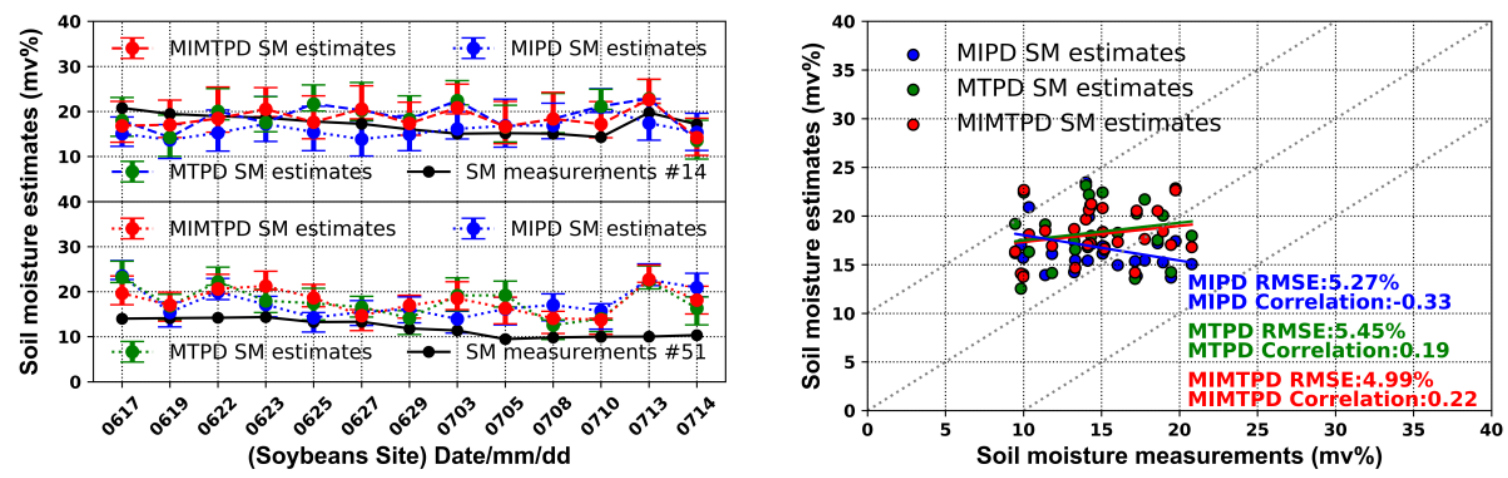

(d) Soybean field 

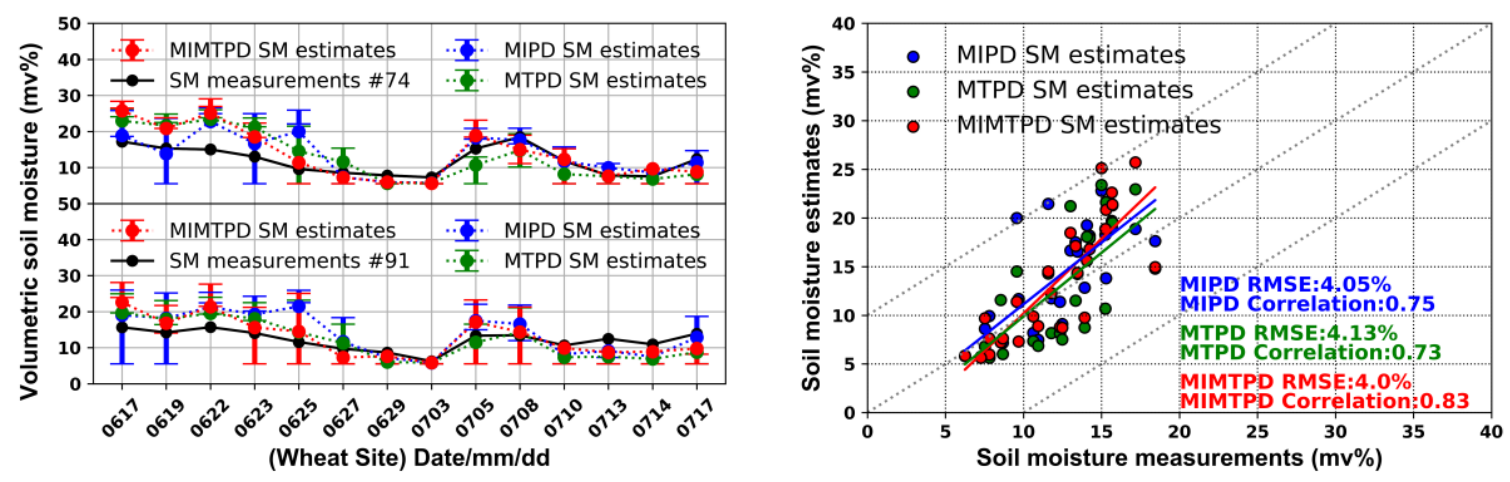

(e) Wheat field
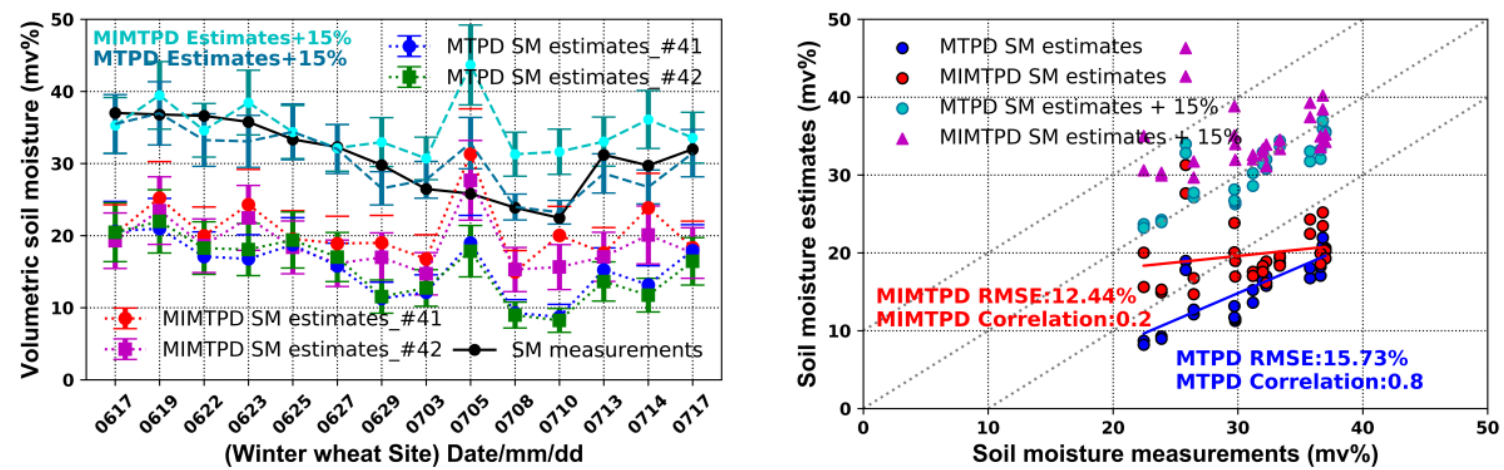

(f) Winter wheat field

Fig. 6. Time series SM estimates from the MIPD, MTPD and MIMTPD methods; variation of site measurements and scatterplots of SM over bean, canola, corn, soybean, wheat and winter wheat fields. (a) Bean field. (b) Canola field. (c) Corn field. (d) Soybean field. (e) Wheat field. (f) Winter wheat field.

As shown in Fig. 6, the SM measurements are stable (maximum change is less than 5\%) through the period between June 17 and July 17, over bean, corn and soybean areas. For canola, wheat and winter wheat fields, the SM measurements present a trend of early decrease and late increase with a dynamic range of approximately 15\%. The MIPD, MTPD and MIMTPD algorithms provide similar retrieval accuracy rates with RMSEs between $2.88 \%$ and $5.45 \%$ over bean, canola, corn, soybean and wheat areas. The performance of multiple PolSAR retrieval methods varies depending on the crop type. For winter wheat, an underestimation of $15 \%$ is found on every observation date despite the high correlation between estimates and measurements (Fig. 6f). The possible influencing factors are explored in Sections 4.3 and 4.5.1. A weak correlation between estimates and measurements can also be found because of the low fluctuation of SM over bean and soybean areas. In canola and wheat areas, the change of SM estimates conforms to the variation of in-situ measurements, hence showing high accuracy and strong correlation. With regard to the similar results of the three SM inversion algorithms, SM maps and histograms along the UAVSAR campaign are collected from MIMTPD methods to investigate the spatial characteristic and inversion rate of SM estimates over part of the selected crop fields (see Fig. 7). It shows an inversion rate of $85 \%-99 \%$ depending on the crop type and observation date. Invalid inversion pixels can also be observed in Fig. 7 because the derived 
soil dielectric constant which equals the lower bound (i.e. $\varepsilon_{\text {soil }}=2$ ) is taken as an invalid estimation when counting the inversion rate in our procedure.
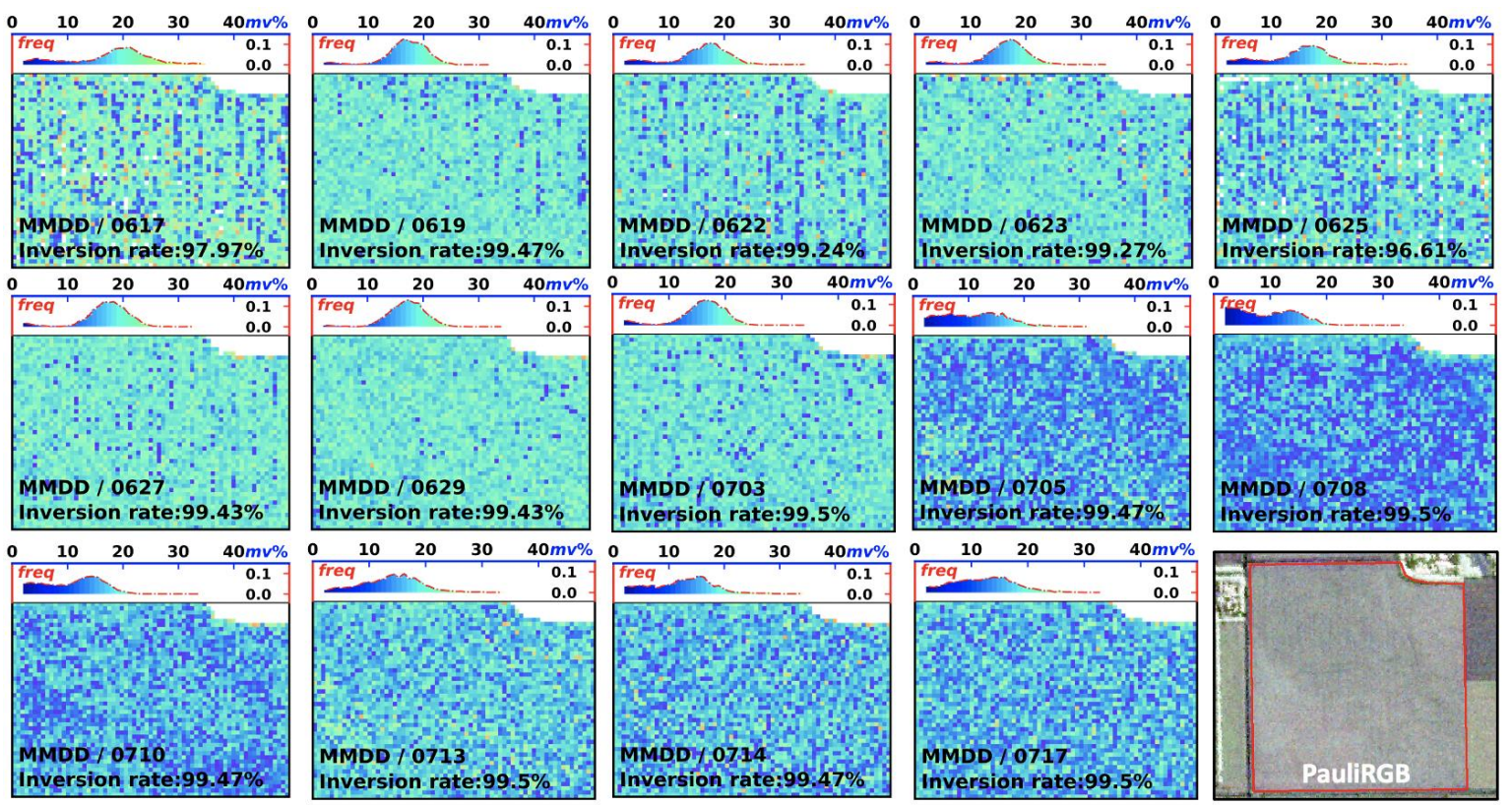

(a) SM map over bean field
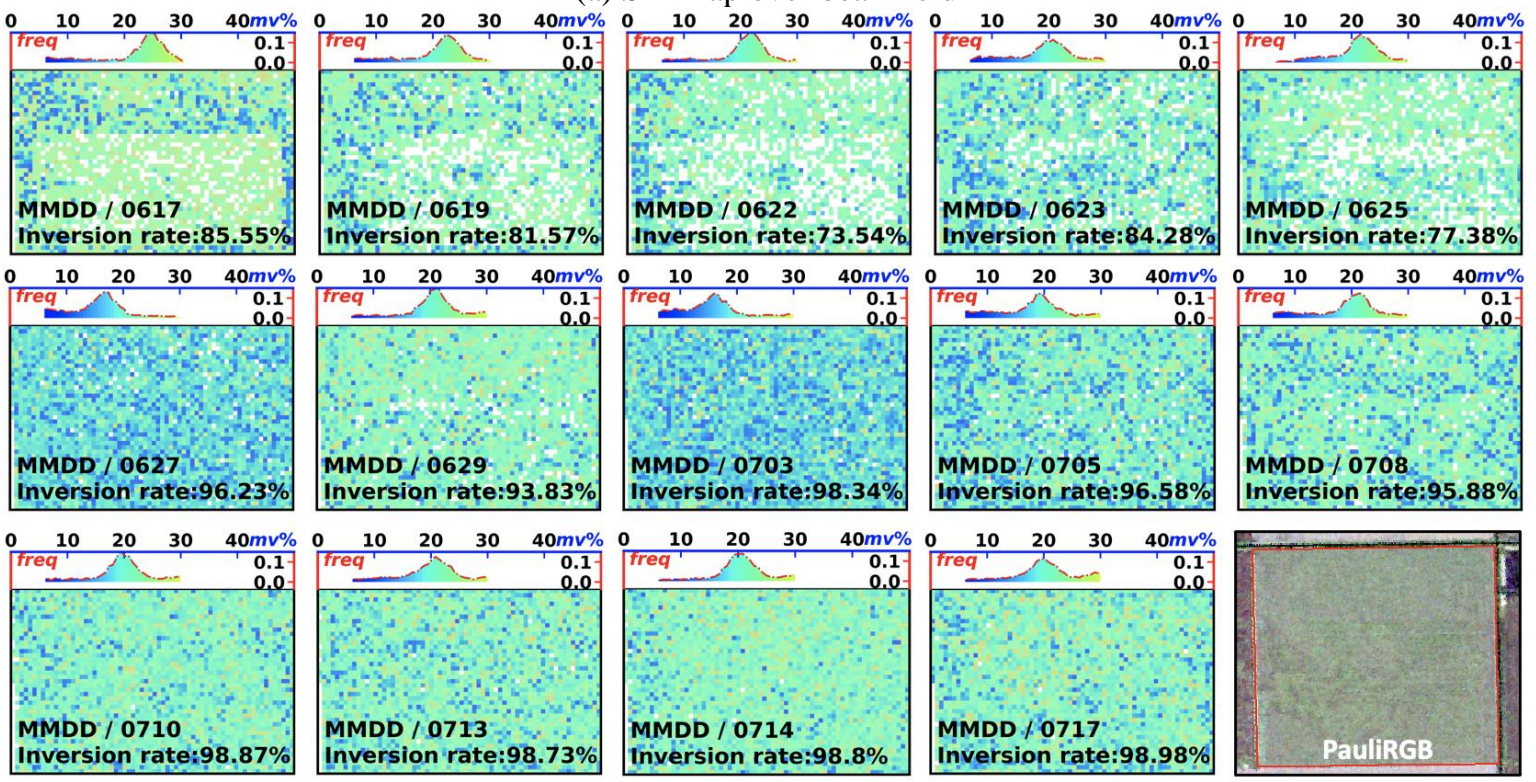

(b) SM map over canola field 

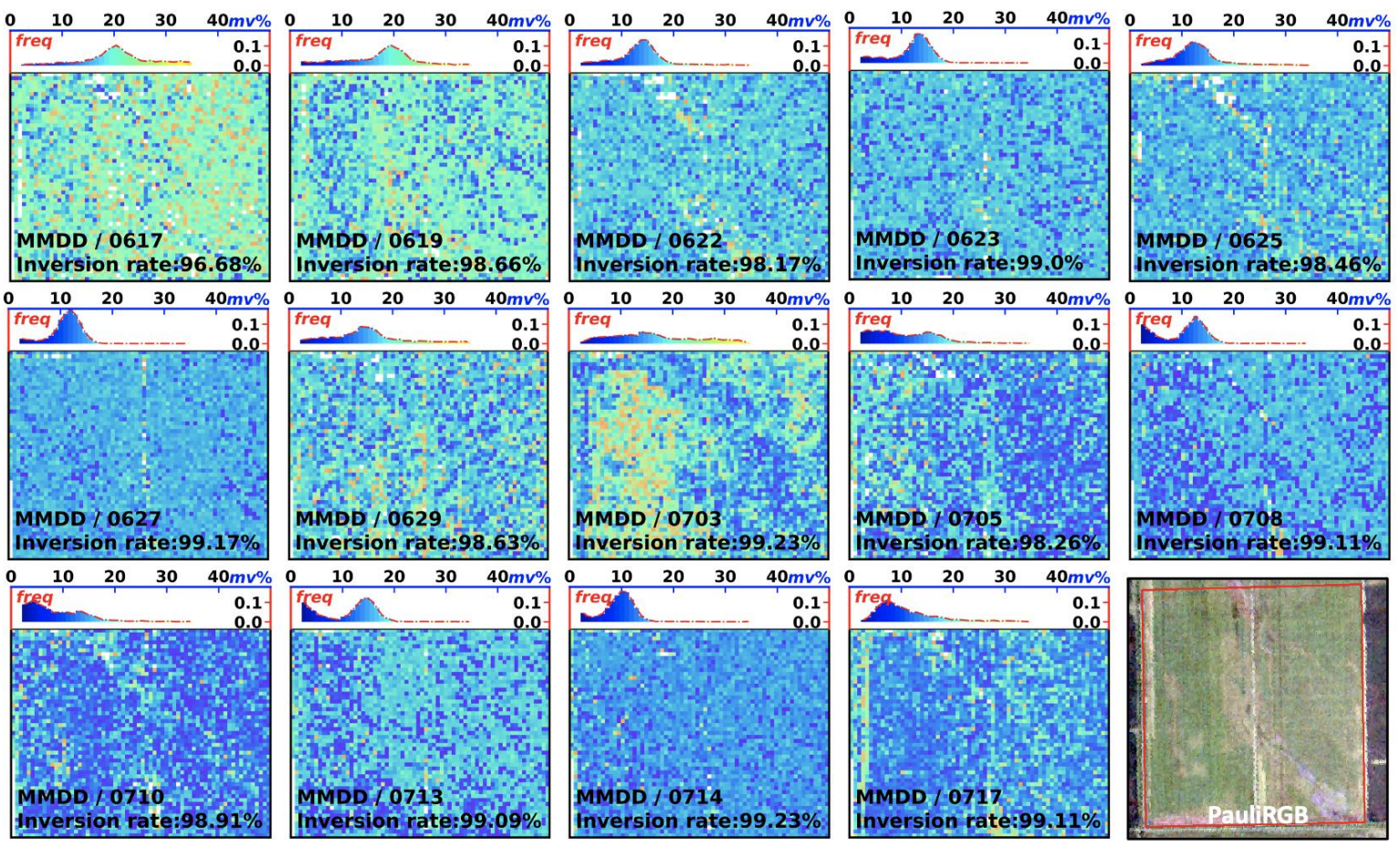

(c) SM map over corn field
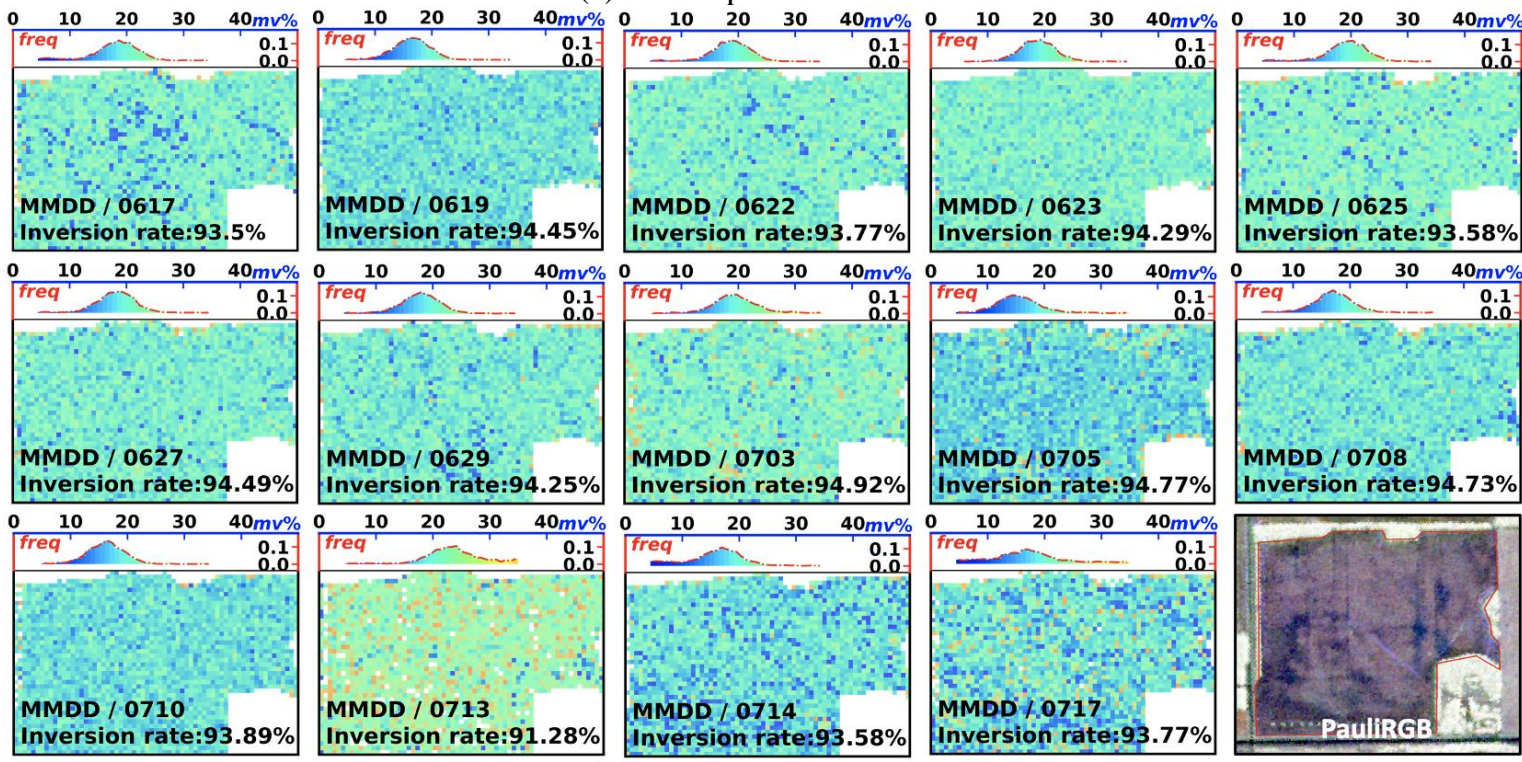

(d) SM map over soybean field

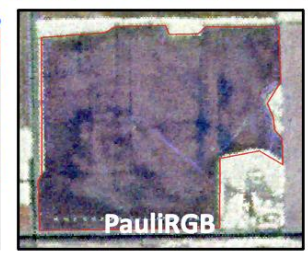




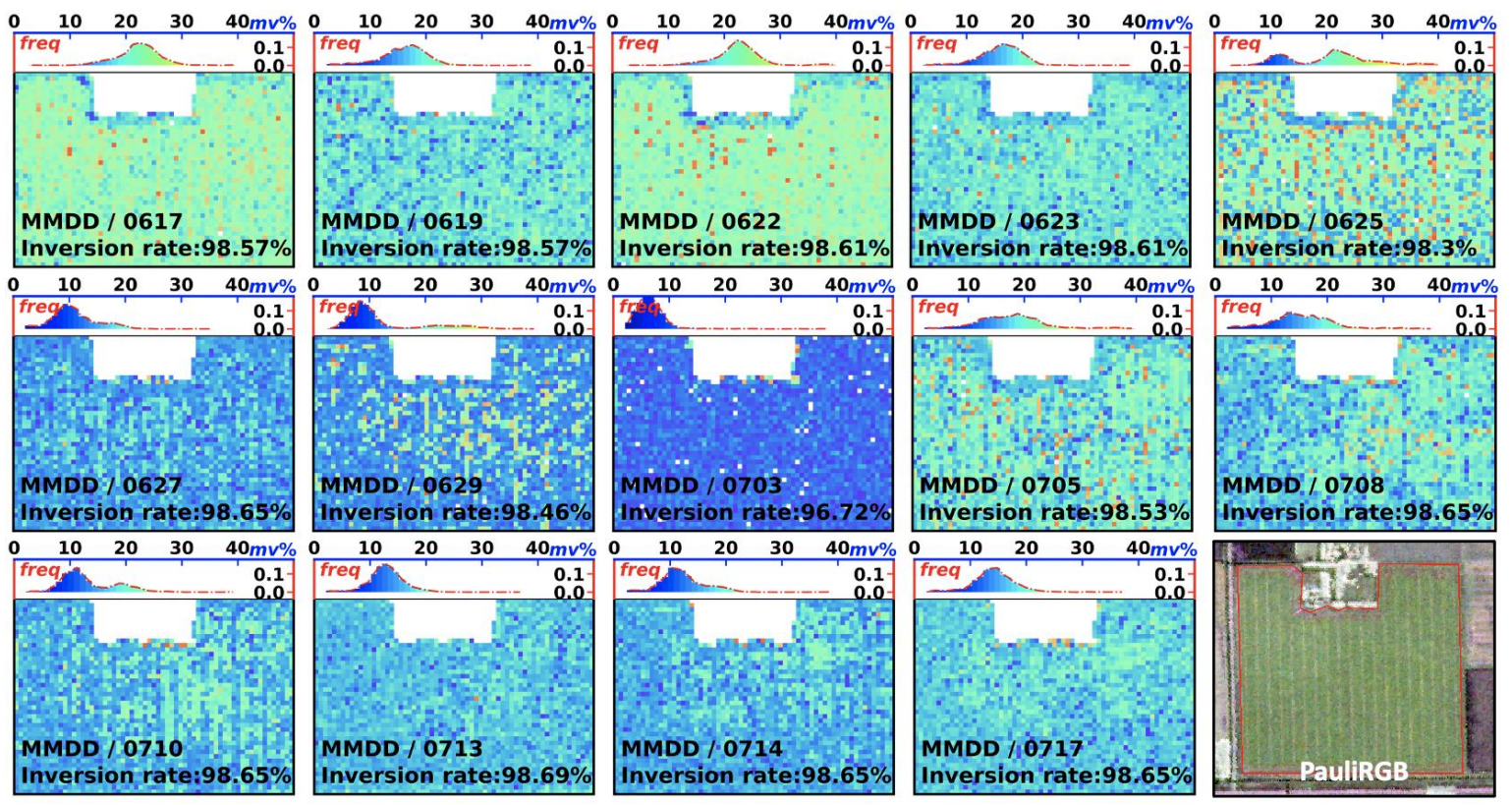

(e) SM map over wheat field

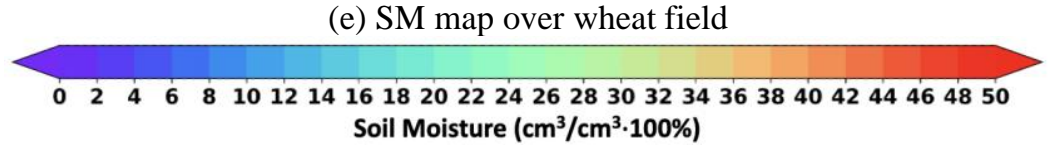

Fig. 7. Map, histogram and inversion rate of estimated SM over selected bean, corn, canola, soybean, and wheat fields. The red polylines in PauliRGB images denote the area of selected crop fields. In the SM map of each subplot, the areas which are outside the polyline and the pixels with invalid estimations which are inside the polylines are all masked in white.

The scatterplots of MIPD, MTPD and MIMTPD SM estimates are individually depicted in Figs. 8a, 8c and 8e. They indicate that all the three methods have similar performance on the SM estimation. The MIPD and MIMTPD methods provide an RMSE of $4.3 \%$ and a correlation coefficient of 0.6 for SM ranging from $5 \%$ to $25 \%$. The performance of the MTPD retrieval method is somewhat better compared with MTPD and MIMTPD results, providing an overall RMSE of $4.21 \%$ and a correlation coefficient of 0.62 . To verify the effectiveness of using GVSM model in our proposed method, we also implement the SM inversion experiment by embedding the volume scattering model of Yamaguchi et al. (2005) into the MIPD, MTPD and MIMTPD retrieval framework. The results show that the GVSM model performs better than the Yamaguchi et al. model on SM retrieval over selected crop fields. Figs. 8b, 8d, and 8f present an RMSE of approximately $8.0 \%$ and a correlation coefficient of around 0.3 when using the model of Yamaguchi et al. in the proposed multiple observation-based SM retrieval framework. 


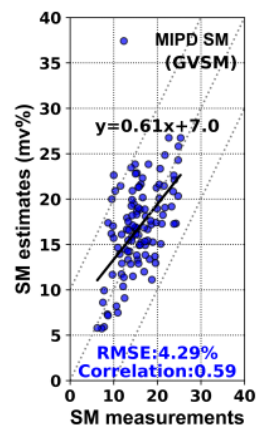

(a)

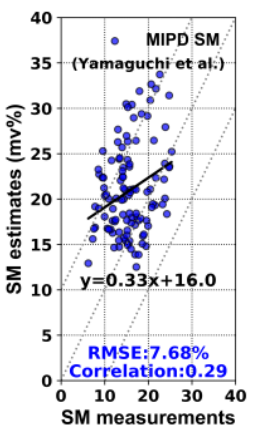

(b)

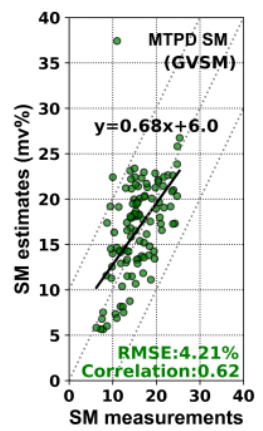

(c)

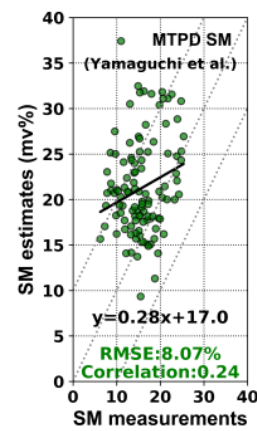

(d)

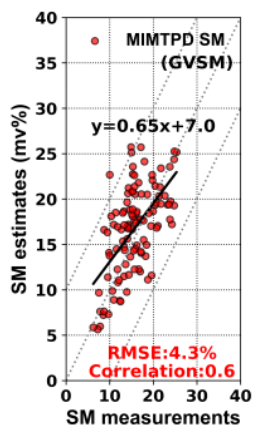

(e)

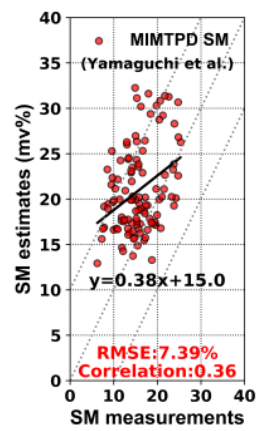

(f)

Fig. 8. Scatterplot, RMSE and correlation coefficients of multi-incidence and multitemporal SM estimates over bean, canola, corn, soybean, and wheat fields. (a) MIPD+GVSM results vs. measurements. (b) MIPD+Yamaguchi results vs. measurements. (c) MTPD+GVSM results vs. measurements. (d) MTPD+Yamaguchi results vs. measurements. (e) MIMTPD+GVSM results vs. measurements. (f) MIMTPD+Yamaguchi results vs. measurements.

\subsection{Crop condition and evolution of the scattering mechanisms}

As illustrated in Fig. 6, the varying performance of different PolSAR SM retrieval algorithms can be seen over different crop types due to many factors, including (i) vegetation structure, PWC and biomass; (ii) the penetration depth of electromagnetic waves and incidence angles; and (iii) the heterogeneity of surface conditions, e.g. soil roughness and correlation length. Here, the evolution of the crop scattering mechanism, i.e. surface, dihedral and volume scattering power ratio which are derived from multiple PolSAR decompositions (Section 2.1) are shown in Fig. 9. In addition, the variation of crop height, diameter, biomass and area PWC collected from in-situ measurements are presented to track the relationship between crop properties and scattering mechanism.

As shown in Fig. 9, for the bean field, although the surface scattering power increases and the volume scattering decreases with the growth of biomass and PWC, the comparable estimates and measurements of SM have no significant change until July 3 (Fig. 6a). However, the SM is underestimated when the dihedral scattering component appear, i.e. from July 5 , which could also be observed over the soybean area. For the canola area, surface scattering increases progressively and becomes clearly dominant, reaching its higher value at the end of the field campaign. Meanwhile the canola height and PWC also peaked on July 17. As shown in Fig. 9b, the evolution of dihedral scattering power ratio seems to be regulated by the crop diameter and SM.

The temporal scattering mechanism profile of the corn field also reveals a tendency of increasing surface scattering and decreasing volume scattering power ratio, which is similar to that of canola before July 5 . At the later campaign stage (July 5-17), the dihedral scattering power ratio is higher than that of the early stage 
due to the increase in corn height, diameter and PWC. As for the SM inversion, the multi-incidence and multitemporal PolSAR retrieval algorithm can present the temporal variation of SM over the corn field.

Compared with that of the canola, corn, and soybean fields, the crop height, biomass and area PWC of wheat varies within a smaller range. Especially, stem diameter does not exhibit any visible change during the entire period of the campaign. Coincidentally, the comparable power ratio of surface and volume scattering component also shows no significant change, whereas the dihedral scattering component seems to be affected by the SM as shown in Figs. 6d and 9d. For winter wheat, although the retrieval performance yields an unexpected underestimation of $15 \%$ for each observation date, the strong correlation $(R=0.8)$ between estimates and measurements reveals a great potential to describe the evolution of SM. Referring to Fig. 9f, we can attribute the underestimation issue for winter wheat to the high area PWC, i.e. nearly $1000 \mathrm{~g} / \mathrm{m}^{2}$ larger than that of other crops.

Although previous studies suggested that the two-component PD method which disregards the dihedral scattering component could greatly reduce the complexity of SM retrieval, a three-component decomposition model is also necessary and more generalised when the ratio of dihedral scattering is larger than $10 \%$ at the end stage of the campaign.
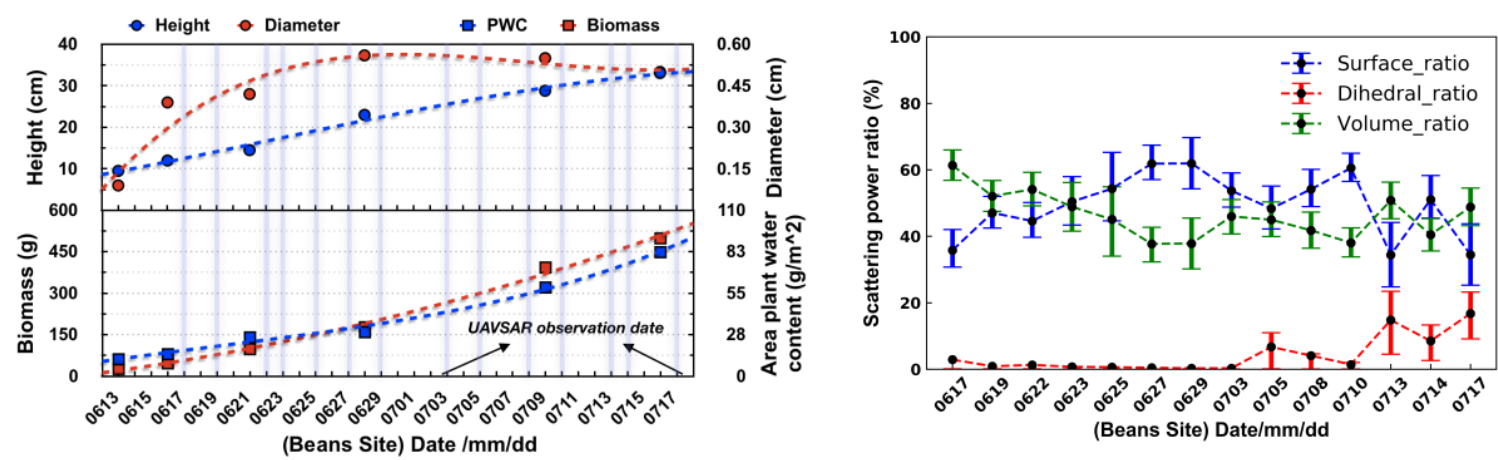

(a) Bean field
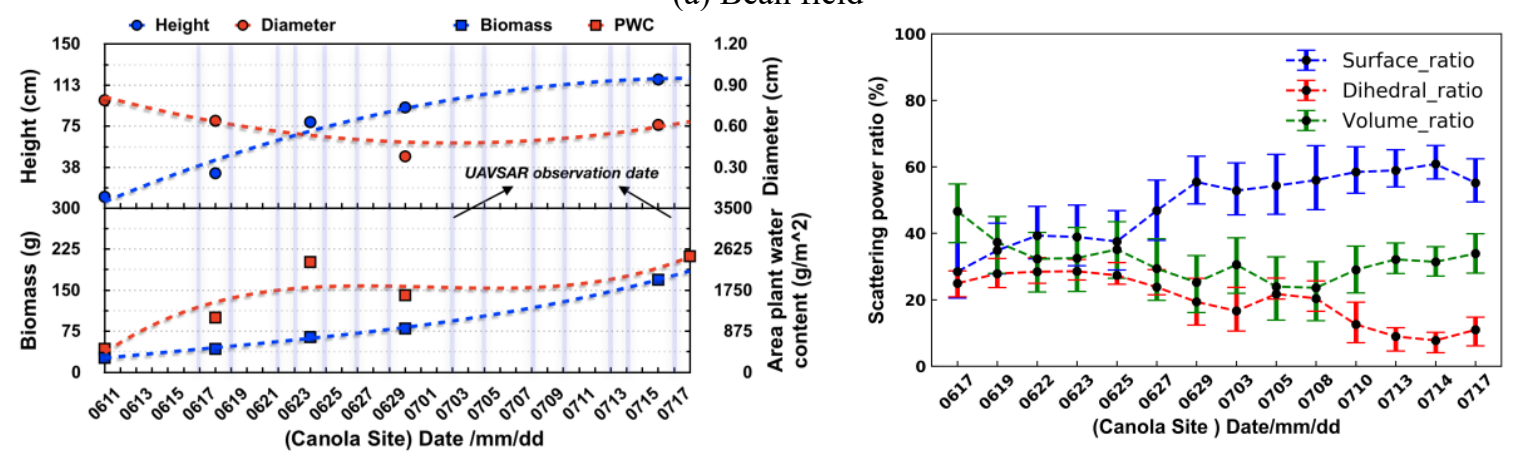

(b) Canola field 

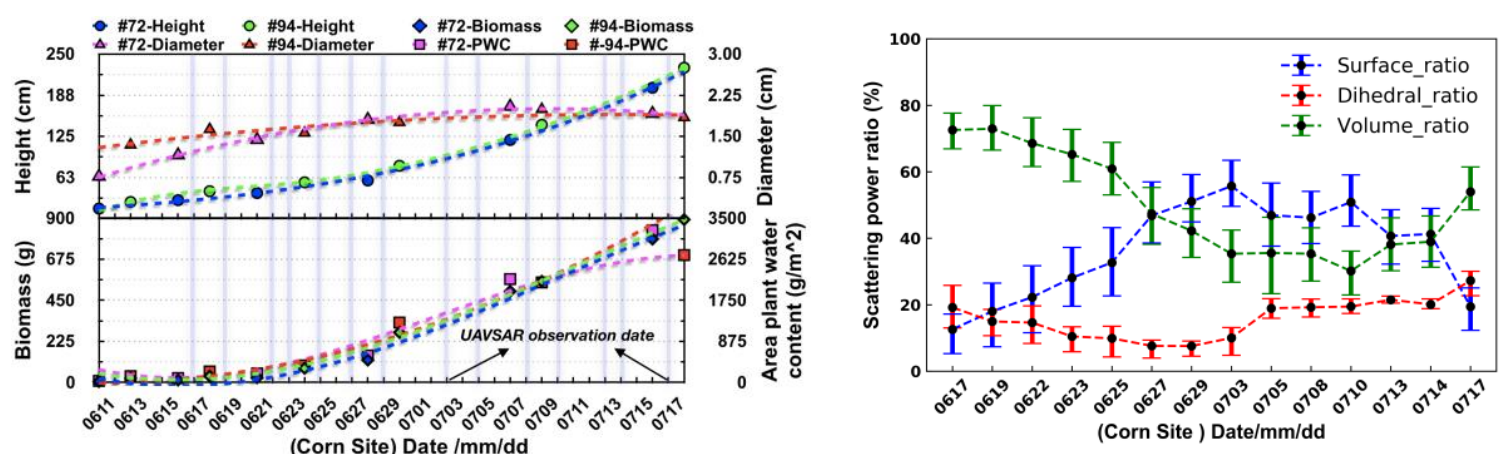

(c) Corn field
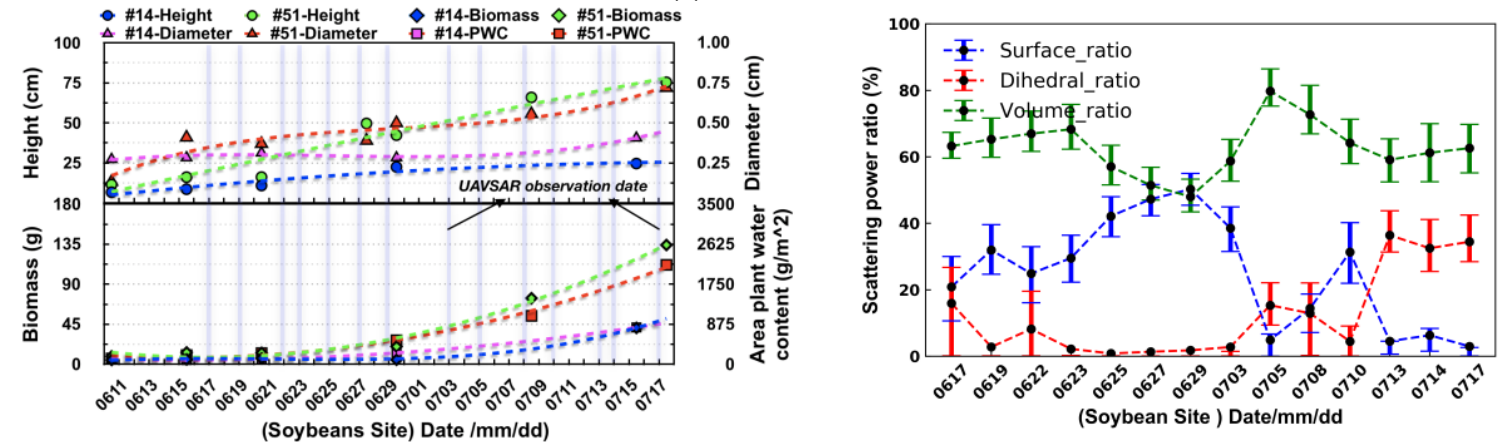

(d) Soybean field
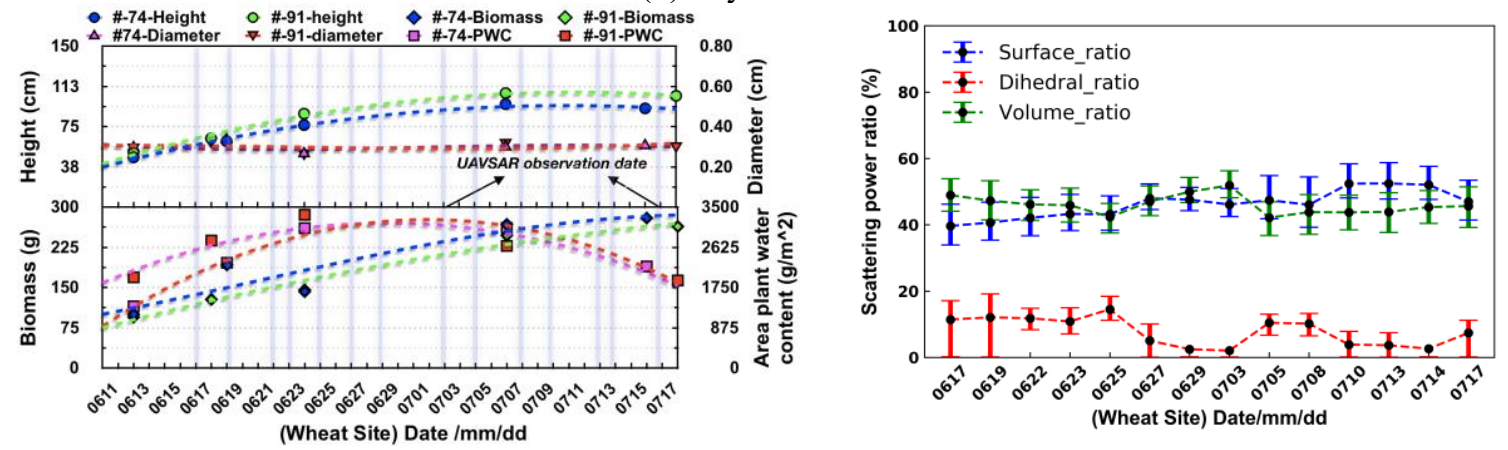

(e) Wheat field
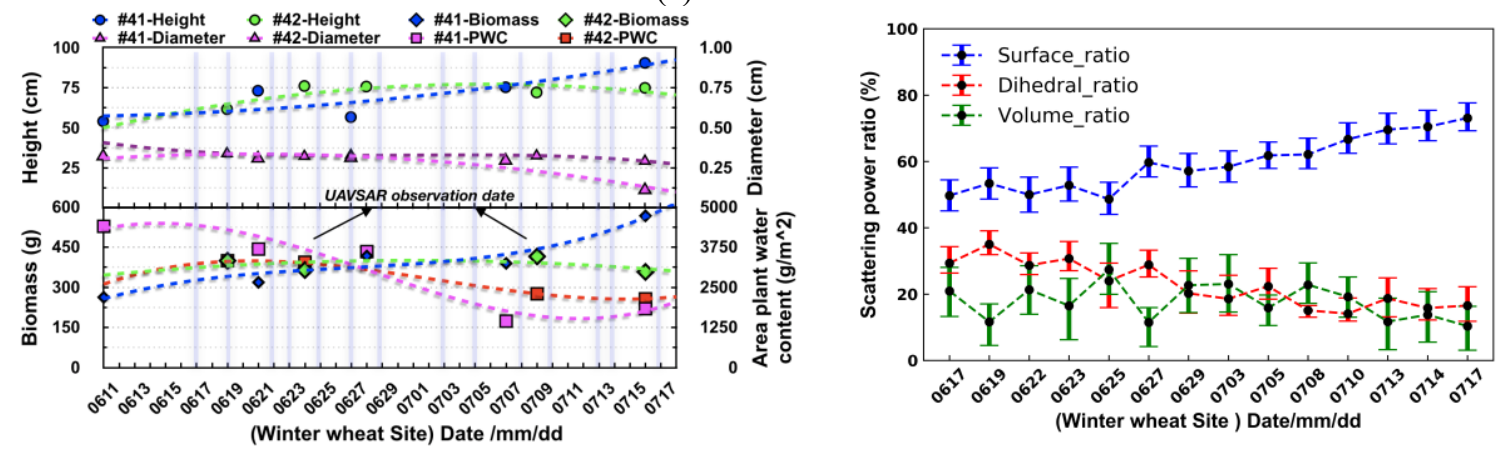

(f) Winter wheat field

Fig. 9. Temporal evolution of crop height, diameter, biomass and area PWC, and the variation of surface, dihedral and volume scattering ratio over bean, canola, corn, soybean, wheat and winter wheat fields.

\subsection{Crop stem permittivity estimation}

According to the proposed SM retrieval framework, i.e. eqs. (12) and (13), the SP $\left(\varepsilon_{t / s}\right)$ could also be simultaneously calculated together with the soil dielectric constant from the extended dihedral scattering model $\left(T_{D_{-} \text {Fresnel }}\right)$. In Fig. 10, we illustrated the map of the estimated SP when dihedral scattering mechanism 
is significant over part of the selected crop fields. It shows that the inversion rate ranges from $40 \%$ to $99 \%$ depending on the crop type and observation date. The inversion rate of SP is very low especially at the beginning of the SMAPVEX12 campaign, which is in accordance with the power ratio of dihedral scattering in Fig. 9a and Fig. 9d. This could be partly explained by the low biomass and area PWC of bean and soybean. However, the low inversion rate could also be observed on June 29 and July 3 because of the low dihedral scattering power ratio over wheat field.

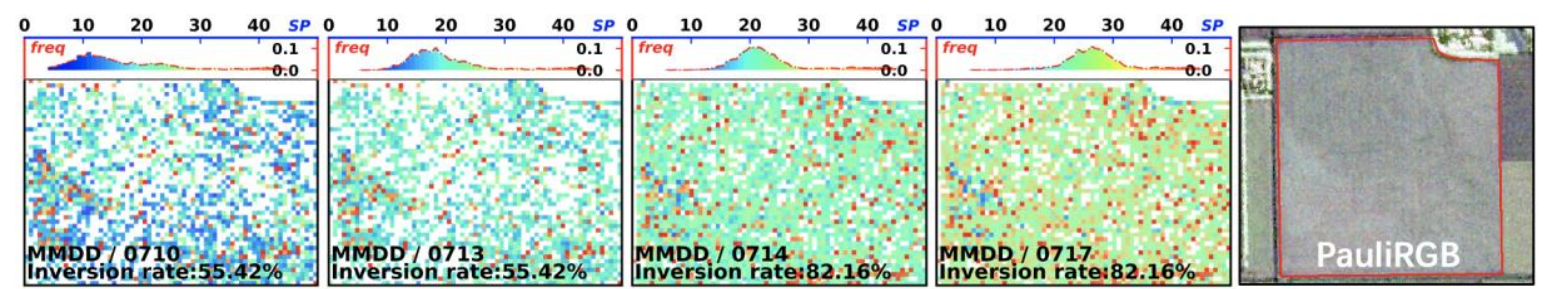

(a) SP map over bean field

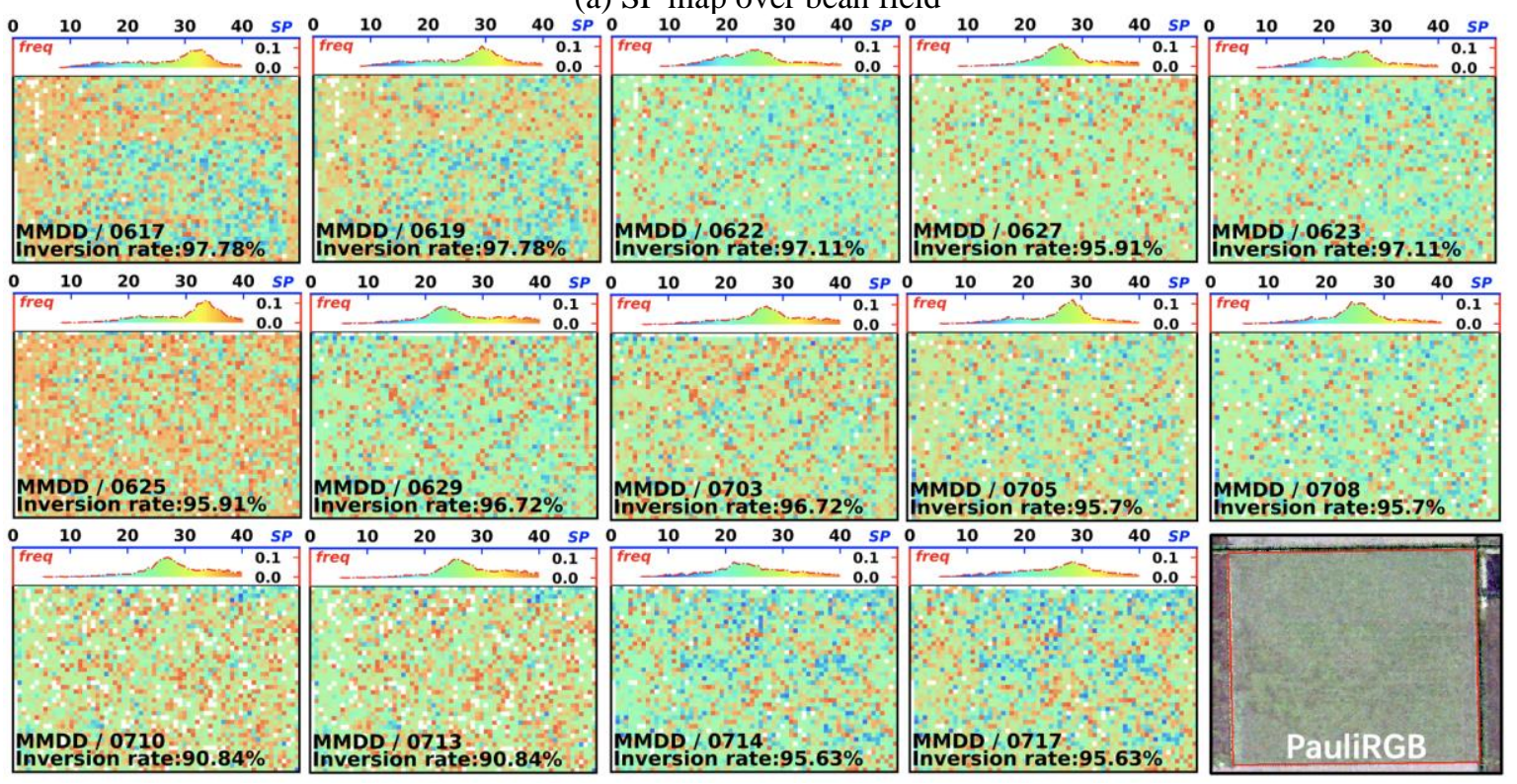

(b) SP map over canola field 

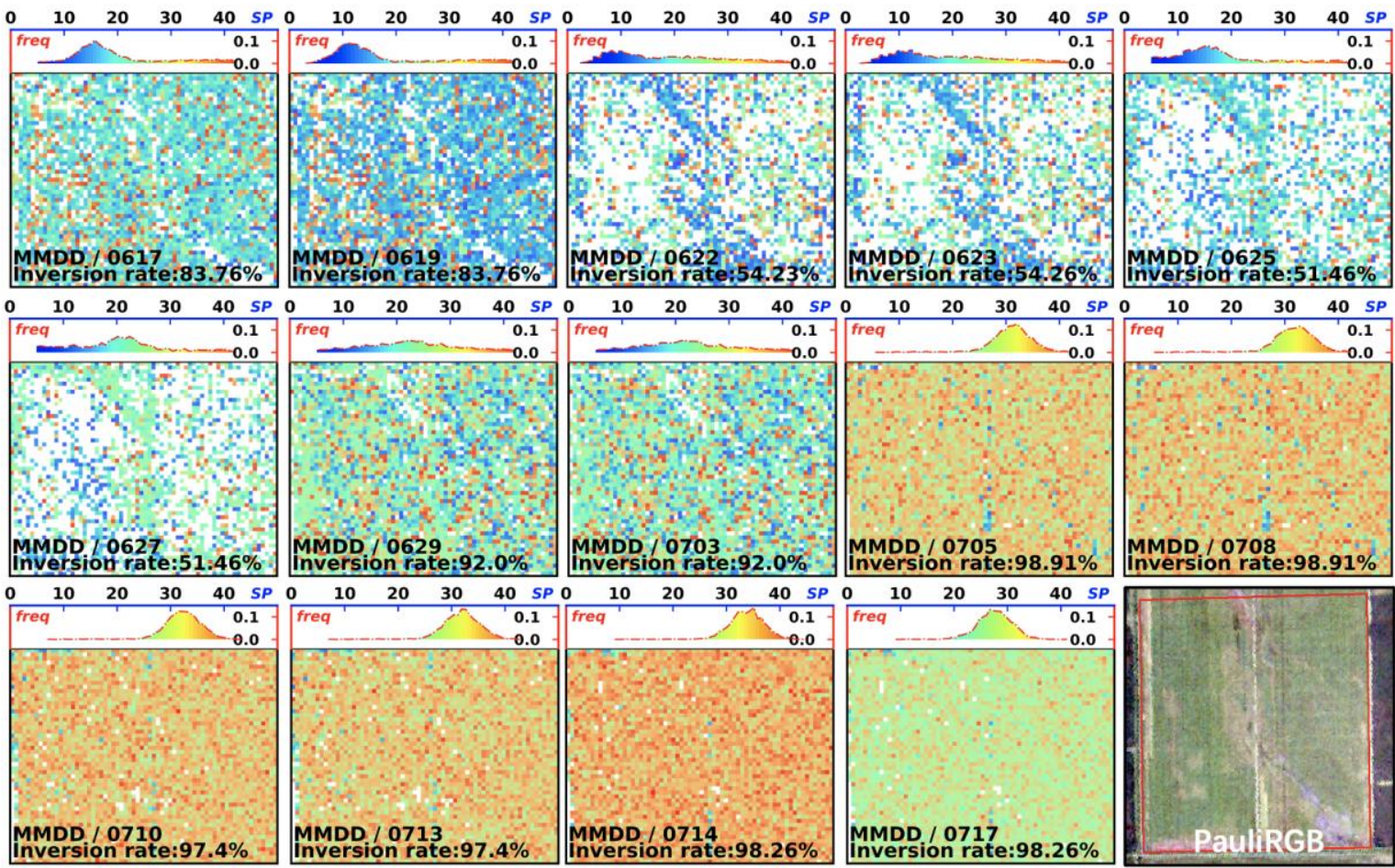

(c) SP map over corn field

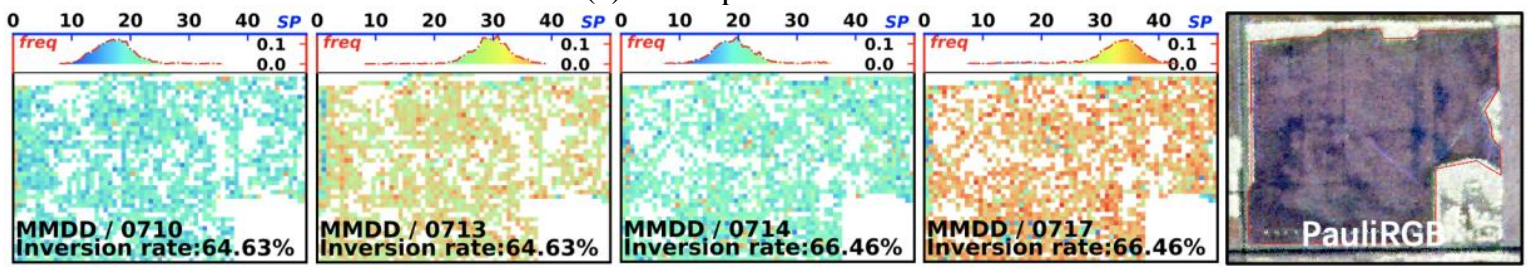

(d) SP map over soybean field

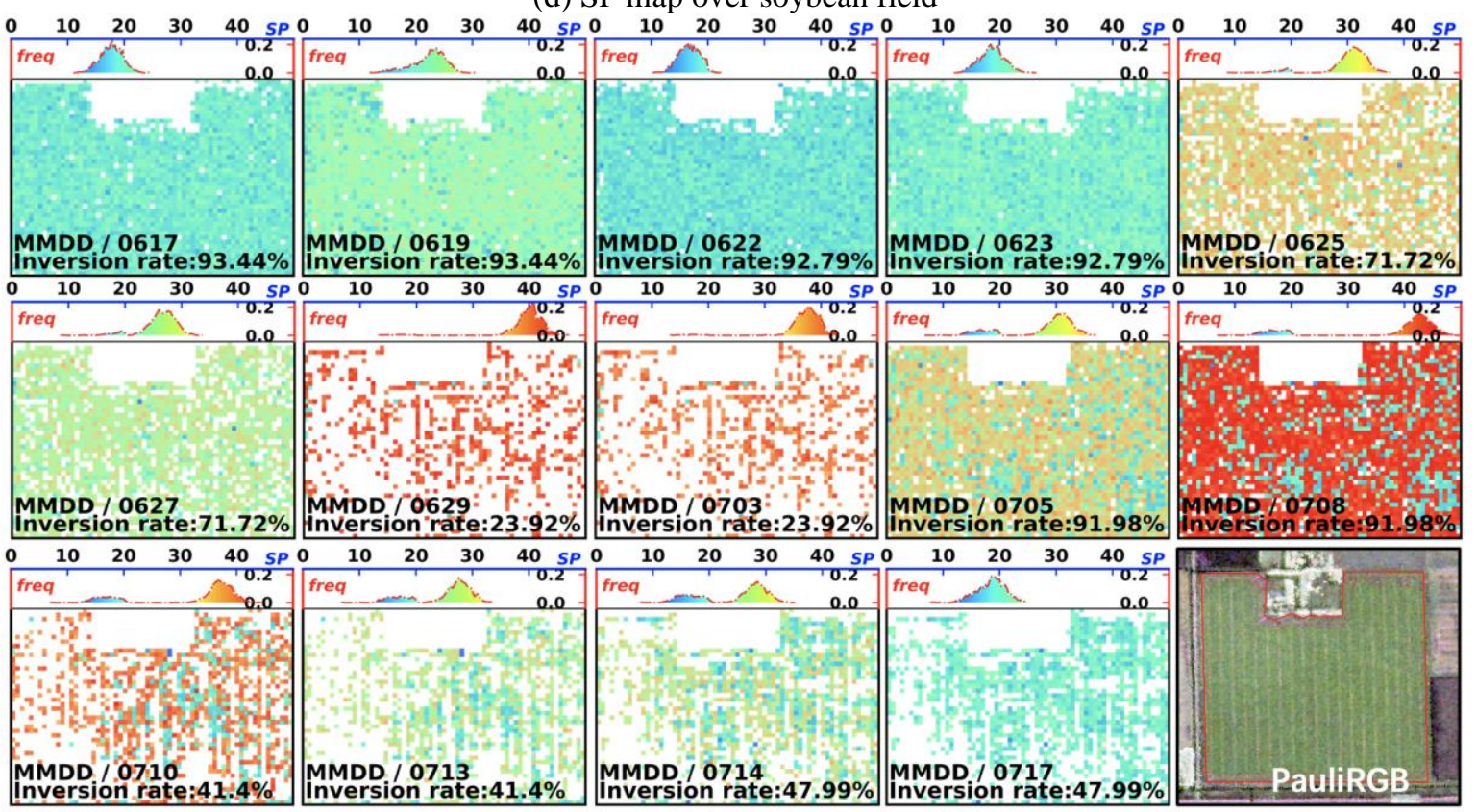

(e) SP map over wheat field

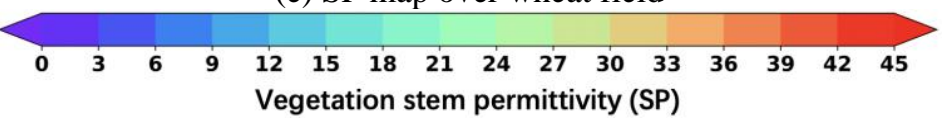

Fig. 10. Map, histogram and inversion rate of estimated SP over bean, corn, canola, soybean and wheat fields.

The temporal variation of retrieved SP over bean, canola, corn, soybean, wheat and winter wheat is 
illustrated in Fig. 11a and 11b. Unlike the variation of estimated SM, the derived SP shows a significant change during the crop growing season, particularly over bean, corn and soybean fields. In addition, the SP presents a low value at the early stage and increases with the growth of crops, which is consistent with the change of area PWC (Fig. 11c and 11d). The relative change of the estimated SP over canola, wheat and winter wheat is similar to that of the area PWC. For winter wheat, the higher area PWC corresponds to the larger value of SP (before July 3) and vice versa (from July 5 to July 17). Over wheat field, the SP first increases and then decreases with the variation of area PWC. In contrast, over canola area, both the PWC and SP tended to remain stable in the observation period.

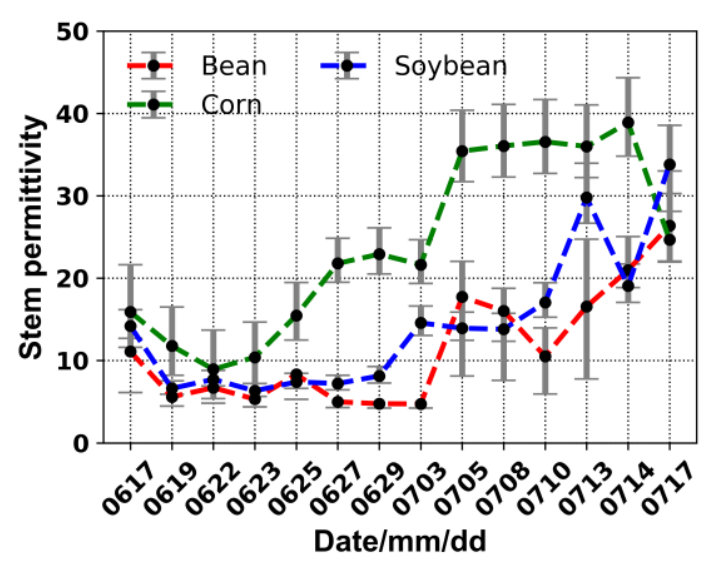

(a)

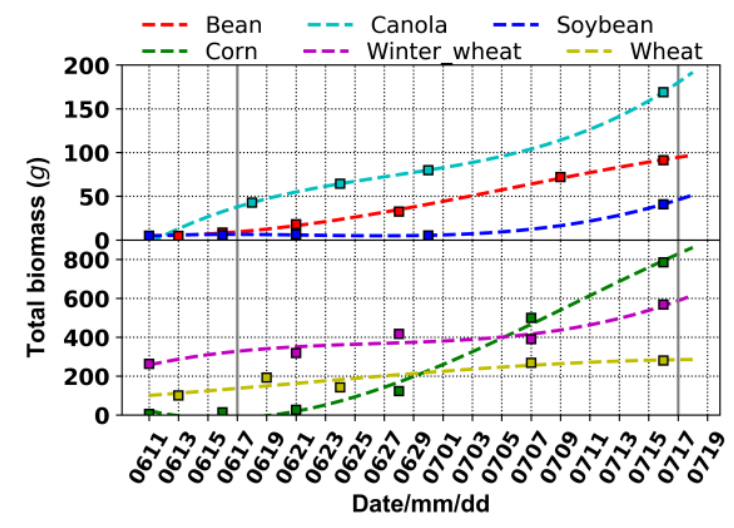

(c)

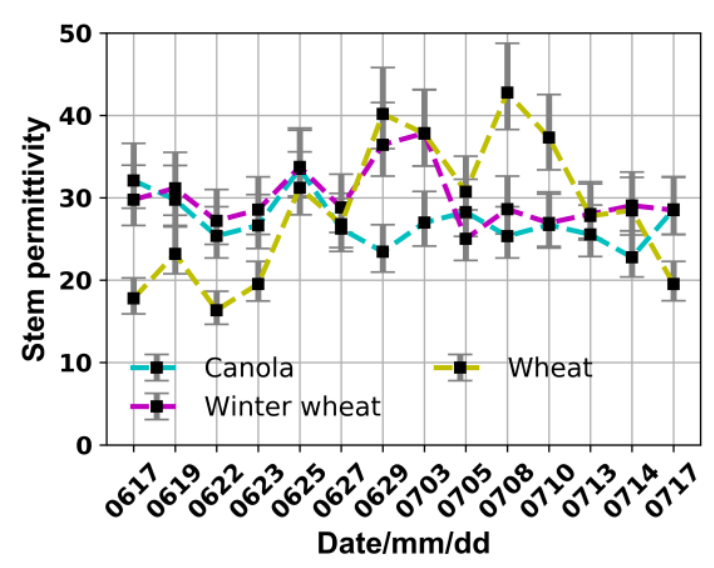

(b)

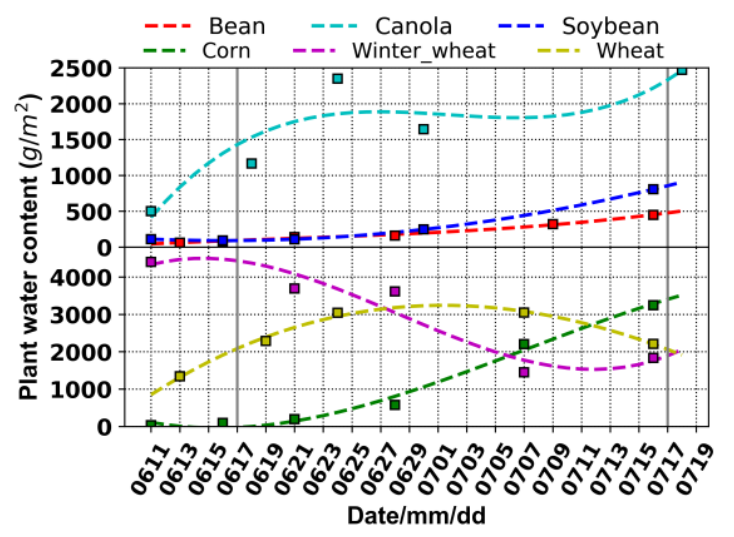

(d)

Fig. 11. Estimation of SP from multiple PDs and the measured vegetation biomass and area PWC. (a) and (b) are the evolution of estimated vegetation SP for bean, soybean, corn, canola, wheat and winter wheat.

(c) and (d) are the measured total biomass and area PWC over the selected crop fields, respectively.

Considering the variation of SM within a short time interval and its influence on the SP, this work also assesses the interaction between SM and SP by using a window time-lagged cross correlation (WTLCC) method. Here, we assume that the change of the PWC is led by the evolution of SM, i.e. soil dielectric constant is selected as 'Signal-1', and the SP is selected as 'Signal-2'. Given 14 PolSAR observations during 
the SMAPVEX12 campaign, we process nine epochs when implementing the WTLCC algorithm. In Fig. 12, the higher cross correlation values are located in the offset area, which equals to -2 or -1 for most epochs. It could be inferred that the estimated time-series SM plays a lead role in changing the estimated SP to some extent (see Fig. 12).
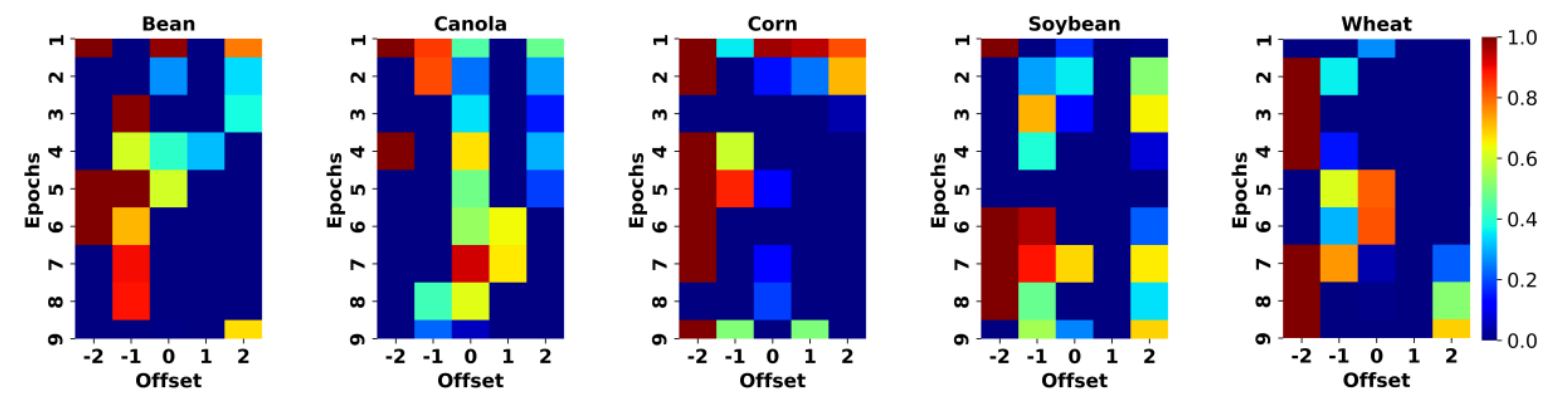

Fig. 12. Rolling window time-lagged cross correlation between SM and SP for continuous windows. The WTLCC method is implemented with window size $=6$; rolling step $=1$; signal-1: soil dielectric constant; signal-2: SP; time lag range $=[-2,2]$.

To investigate the physical meaning of the estimated SP, we compare the SP estimates with the total biomass and area PWC. Fig. 13 shows the scatterplots of the SP, total biomass and PWC over selected crop fields. A positive linear relationship can be observed between SP and biomass (red line), as well as SP and PWC (blue line), particular for bean, corn, soybean and wheat. For example, over soybean fields the correlation between SP and biomass is 0.89 , whereas the correlation of SP and PWC is valued at 0.92 . However, over winter wheat fields, negative or no significant linear relationship between SP, biomass and PWC is found because of the inaccurate estimation of SM induced by the empirically zero co-pol phase difference $(\Delta \varphi)$ in the scattering model (Hajnsek et al., 2009; Jagdhuber, 2016) and the high value of crop biomass (>300 g) and PWC (close to $4500 \mathrm{~g} / \mathrm{m}^{2}$ ). The influence of $\Delta \varphi$ on SM estimation is analysed in Section 4.5.1 of this paper.
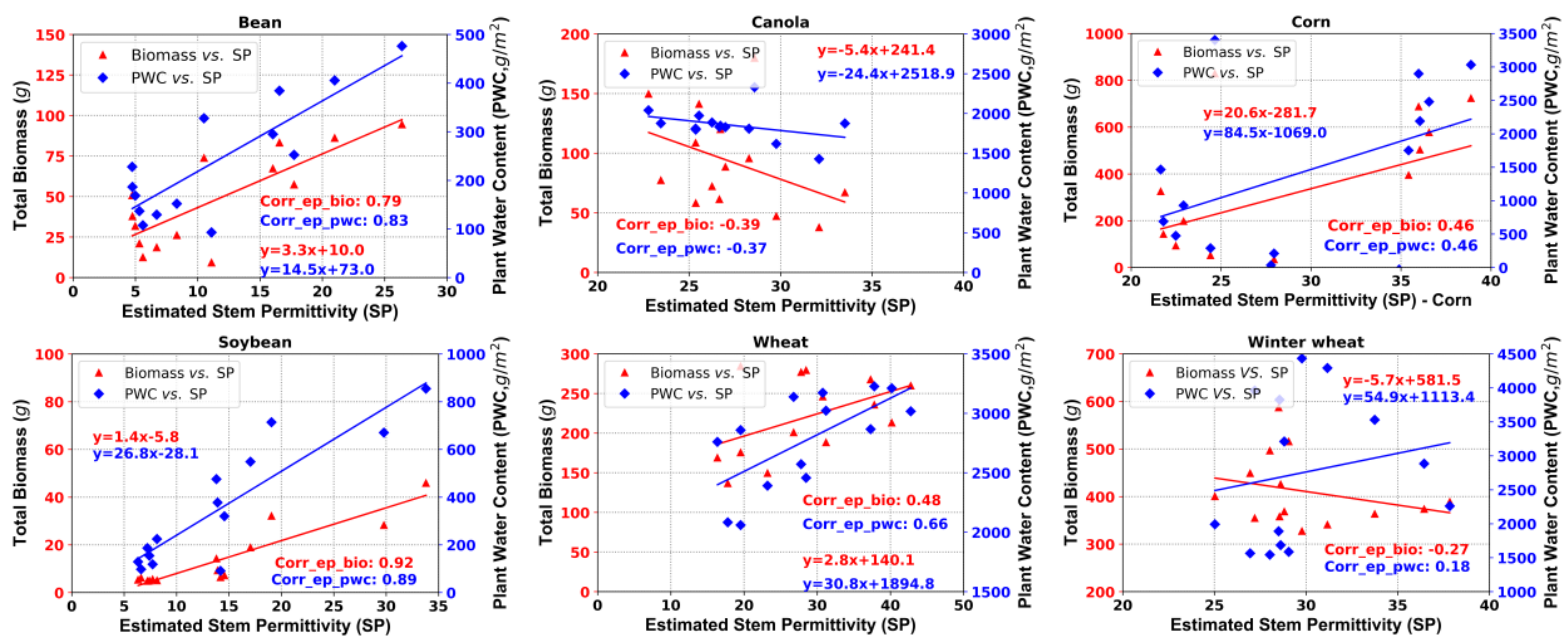
Fig. 13. Scatterplot of the SP, total biomass and area PWC over bean, canola, corn, soybean, wheat and winter wheat fields.

4.5. Influence of co-pol phase difference and surface depolarisation angle on SM estimation

As mentioned above, SM estimation is inherently affected by the crop type and phenology. In this part, two parameters, i.e. co-pol phase difference $(\Delta \varphi)$ which is related with the crop condition and surface depolarisation angle $(\delta)$ which is dominated by surface roughness, are considered for exploring the possible way to improve the retrieval performance of SM estimation. To investigate the influence of $\Delta \varphi$ and $\delta$ on SM estimation, different values of $\Delta \varphi$ and $\delta$ are introduced in the MT version of the proposed inversion model.

\subsubsection{Soil moisture estimation from different $\Delta \varphi$}

Considering that an underestimation of $15 \%$ is found over the winter wheat field, we use different values of $\Delta \varphi\left(10^{\circ}, 20^{\circ}, 35^{\circ}, 50^{\circ}, 65^{\circ}\right.$ and $\left.85^{\circ}\right)$ in the dihedral scattering model to investigate its influence on SM retrieval performance for winter wheat. Fig.14 illustrates the retrieval accuracy of the SM using different $\Delta \varphi$. The results show that the retrieval accuracy is improved, and the underestimation problem can be solved to some degree by using high values of $\Delta \varphi$. The correlation between the SM estimates and measurements is also improved when $\Delta \varphi=50^{\circ}$, whereas it decreases to 0.3 when $\Delta \varphi=85^{\circ}$. This result could be explained by the fact that a fixed $\Delta \varphi$ may lead to the issue of overestimation on different crop phenology. Although the highest correlation is observed at $\Delta \varphi=35^{\circ}$, the result still poses approximately $12 \%$ underestimation because of the high value of biomass (>300g) and PWC (close to $4500 \mathrm{~g} / \mathrm{m}^{2}$ ) over the winter wheat field. In consideration of the RMSE and correlation coefficient of the SM estimates, the best performance over the winter wheat field is presented at $\Delta \varphi=65^{\circ}$.

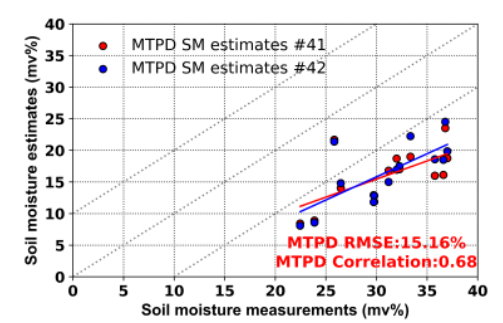

$\Delta \varphi=10^{\circ}$

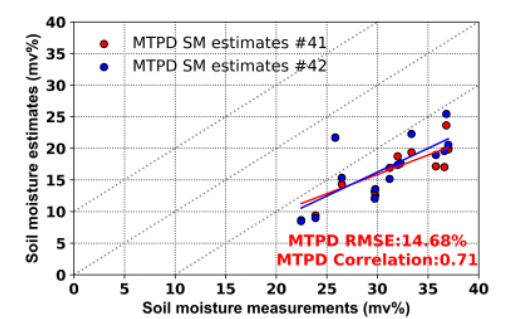

$\Delta \varphi=20^{\circ}$

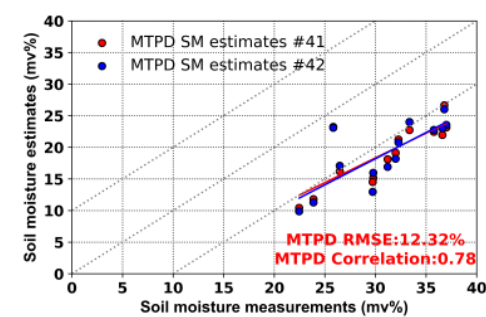

$\Delta \varphi=35^{\circ}$ 


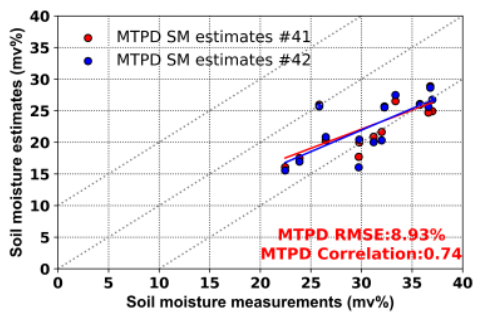

$\Delta \varphi=50^{\circ}$

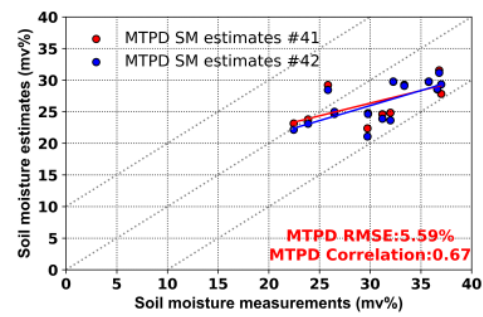

$\Delta \varphi=65^{\circ}$

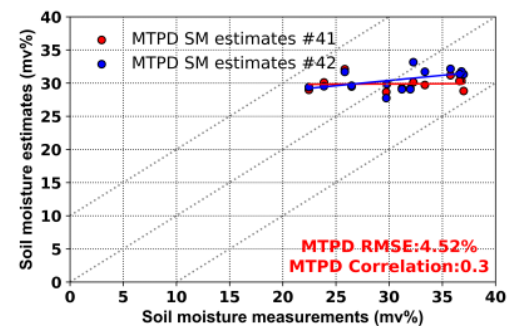

$\Delta \varphi=85^{\circ}$

Fig. 14. SM estimation over winter wheat fields by using different values of $\Delta \varphi$ in the dihedral scattering model.

Inspired by the above findings, we also test the influence of different $\Delta \varphi\left(0^{\circ}, 20^{\circ}, 35^{\circ}\right.$ and $\left.50^{\circ}\right)$ on $\mathrm{SM}$ retrieval over bean, corn, canola, soybean and wheat fields. The results are presented in Table 2. The correlation and accuracy are influenced by $\Delta \varphi$, and the best retrieval performance of $\Delta \varphi$ varies depending on crop type. For instance, the best performance is observed at $\Delta \varphi=20^{\circ}$ for bean and wheat fields but at $\Delta \varphi=0^{\circ}$ and $\Delta \varphi=35^{\circ}$ for corn, canola and soybean. In contrast to winter wheat, no significant difference is found between the RMSE of SM estimates at different values of $\Delta \varphi\left(0^{\circ}, 20^{\circ}, 35^{\circ}\right.$ and $\left.50^{\circ}\right)$ over bean, corn, canola, soybean and wheat fields. The difference in optimum $\Delta \varphi$ between wheat and winter wheat is attributable to the large crop biomass ( $>300 \mathrm{~g}$ ) and PWC (close to $4500 \mathrm{~g} / \mathrm{m}^{2}$ ) over the winter wheat fields; this factor leads to a higher $\Delta \varphi$ than that of wheat. As shown in Fig. 9, the scattering power ratio also indicates that the dihedral scattering of winter wheat is higher than that for wheat field.

\section{Table 2}

Best performance of $\Delta \varphi$ on SM estimation over different crop fields.

\begin{tabular}{cccc}
\hline Crop type & $\Delta \varphi$ & Accuracy (RMSE) & Correlation (R) \\
\hline Bean & $20^{\circ}$ & $2.84 \%$ & 0.17 \\
Corn & $0^{\circ}$ & $3.96 \%$ & 0.64 \\
Canola & $0^{\circ}$ & $2.85 \%$ & 0.64 \\
Soybean & $0^{\circ}$ & $5.45 \%$ & 0.19 \\
& $35^{\circ}$ & $4.73 \%$ & 0.09 \\
Wheat & $20^{\circ}$ & $3.34 \%$ & 0.76 \\
Winter wheat & $65^{\circ}$ & $5.59 \%$ & 0.67 \\
\hline
\end{tabular}

\subsubsection{Soil moisture estimation from different $\delta$}

Fig. 15 illustrates the scatterplots of the surface depolarisation angle and the RMS height of soil on different observation dates, indicating that $\delta$ has a significant linear relationship with RMS height, especially at the beginning of the SMAPVEX12 campaign. However, the correlation between RMS height and $\delta$ also depends on the crop type. For instance, the sensitivity of $\delta$ on RMS height over wheat fields (red dotted line in Fig. 15) is stronger than those over all crop fields (black dotted line in Fig. 5). Furthermore, 
the correlation weakens with the evolution of time and the crop phenology. In other words, the derived $\delta$ is influenced by vegetation structure, as well as biomass and PWC, and could not purely represent the surface roughness information. In this case, it could be inferred that the inaccurate estimated surface depolarisation angle $(\delta)$ also reduces the SM inversion accuracy, especially at the crop growth stage with high PWC and biomass.
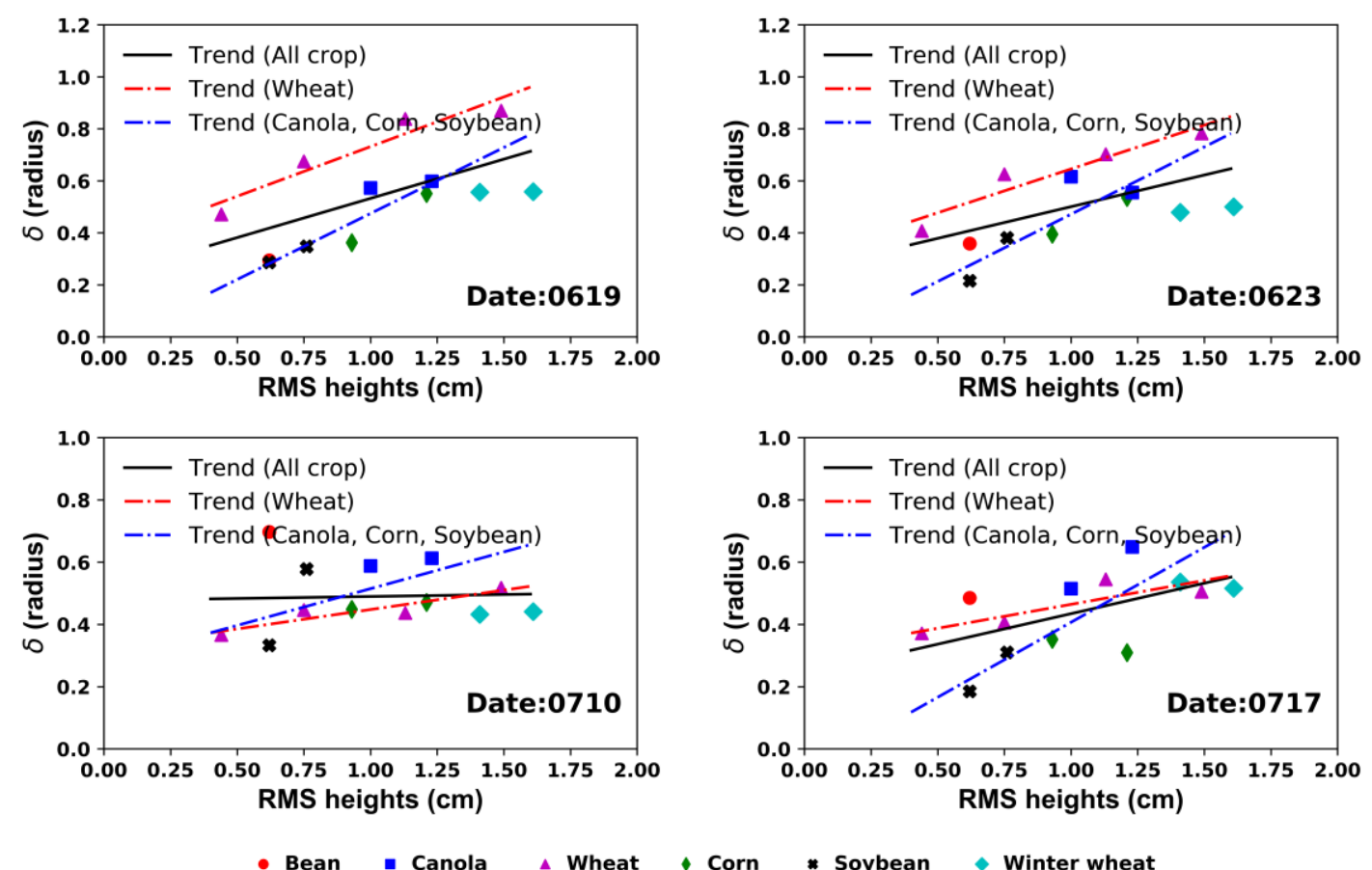

Fig. 15. Relationship between the derived surface depolarisation angle and the RMS height of bean, corn, canola, soybean, wheat and winter wheat fields.

The temporal distribution of $\delta$ over different crop fields is shown in Fig. 16. For bean, corn and soybean fields, $\delta$ changes with the variation of crop parameters and scattering mechanisms. The derived $\delta$ is limited between $10^{\circ}$ and $20^{\circ}$ at the early stage and then increases to nearly $50^{\circ}$ at the middle stage. For canola, wheat, and winter wheat, $\delta$ is higher than $20^{\circ}$ and remains stable during the entire period. Thus result indicates that the derived empirical depolarisation angle also depends on the crop type, structure and phenology. As demonstrated in Fig. 16, the correlation could be observed between the depolarisation angle and crop diameter.
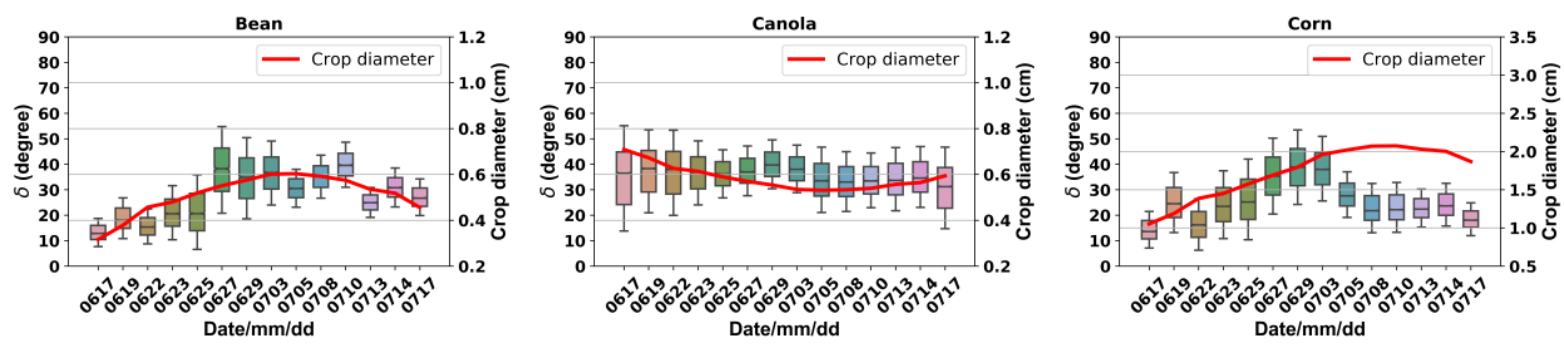

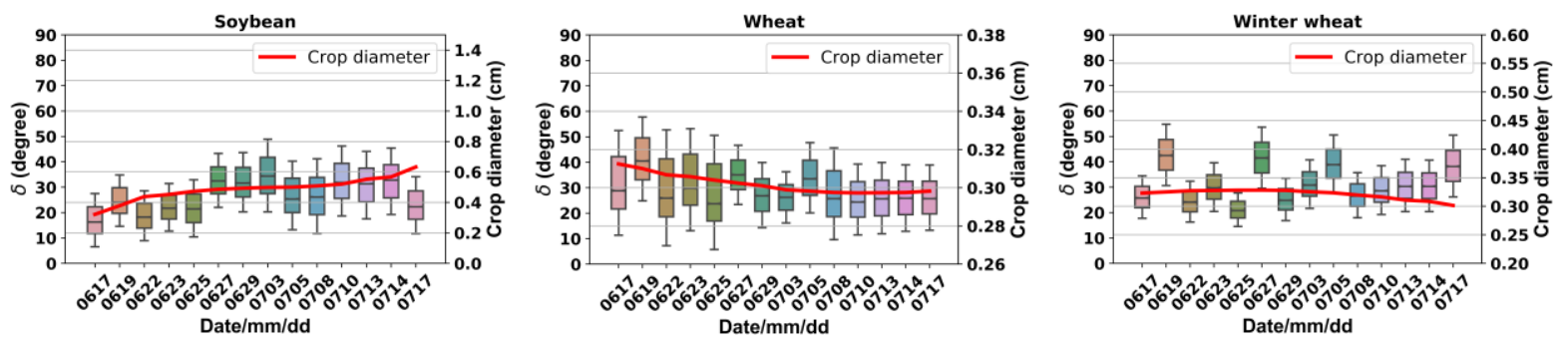

Fig. 16. Temporal variation of soil roughness angle derived from polarimetric circular coherence.

Given that the derived surface depolarisation angle might be attenuated by the canopy scattering, using the PolSAR observations obtained on the least vegetated date to calculate $\delta$ (i.e., $\delta_{\text {least_veg }}$ ) is reasonable. Therefore, to analyse the influence of $\delta$ on SM retrieval, the retrieval performance with different values of $\delta$ are illustrated in Table 3, indicating that accuracy could be improved by using $\delta_{\text {least_veg }}$ in the proposed retrieval model regarding the RMSEs. The correlation between SM estimates and measurements is increased by $\delta_{\text {least_veg }}$ over bean and canola but is decreased over corn, wheat and winter wheat fields possibly because of the influence of vegetation cover on the $\delta_{\text {least_veg }}$ calculation. Especially for winter wheat, it has already reached the reproductive or maturity stage at the beginning of the SMAPVEX12 campaign.

Table 3

Performance of different $\delta_{\text {date } i}$ and $\delta_{\text {least_veg }}$ on SM estimation over different crop fields.

\begin{tabular}{|c|c|c|c|c|}
\hline \multirow{2}{*}{ Crop type } & \multicolumn{2}{|c|}{$\delta=\delta_{\text {date } i}$} & \multicolumn{2}{|c|}{$\delta=\delta_{\text {least veg }}$} \\
\hline & RMSE & $\mathbf{R}$ & RMSE & $\mathbf{R}$ \\
\hline Bean & $3.19 \%$ & 0.04 & $2.5 \%$ & 0.25 \\
\hline Corn & $3.96 \%$ & 0.64 & $4.33 \%$ & 0.56 \\
\hline Canola & $2.85 \%$ & 0.64 & $2.81 \%$ & 0.7 \\
\hline Soybean & $5.45 \%$ & 0.19 & $5.2 \%$ & 0.18 \\
\hline Wheat & $4.13 \%$ & 0.73 & $3.74 \%$ & 0.68 \\
\hline Winter wheat & $5.59 \%$ & 0.67 & $5.02 \%$ & 0.51 \\
\hline
\end{tabular}

\section{Discussion}

PD theorems based on single PolSAR observation have been utilised to estimate SM literatures (Hajnsek et al. 2009; Jagdhuber et al. 2013; Huang et al. 2016a; Wang et al., 2017). The DoIs of the new PD framework indicate that estimating additional unknown parameters, e.g. crop stem permittivity, is possible with PolSAR measurements made at different times and incidence angles. On the basis of our experimental results, the potential of the suggested PD framework in surface SM estimation is observed over agricultural fields. Although the DoIs of MI, MT and MIMT PolSAR observations are larger than that of one single PolSAR observation, they are less than eight (7.8-8.0). Theoretically, the proposed framework remains an underdetermined system. However, the simulations of $f_{s}$ and $f_{d}$ indicate that the number of the unknown parameters in MIPD, MTPD and MIMTPD SM retrieval models could be possibly less than eight. In addition, 
the unknown parameters could be obtained from an underdetermined system by solving the multivariable nonlinear equations.

The same UAVSAR dataset has also been exploited with PD theorem-based retrieval algorithms in a set of fields of the SMAPVEX12 campaign. As shown in Wang et al. (2016, 2017, 2019), the RMSE of SM estimates ranged from $6.1 \%$ to $12.0 \%$ for canola, corn, and wheat, depending on the crop type and vegetation characteristics. Comparing our results (i.e. RMSE between 2.88\% and 5.59\%) with the outcomes of these studies, we find that the inclusion of MI and/or MT PolSAR observations provides some improvement in SM estimation over the selected crop fields of the SMAPVEX12 campaign.

Although the proposed method provides an accuracy of RMSE $\leq 6.0 \%$, a moderate correlation is observed between the estimated and measured SM. This result could be partially attributed to the low dynamic change (15.0\%) of SM during the entire period of the campaign. This work also finds that the variation of estimated SP follows the temporal evolution of area PWC and biomass, especially for bean, corn and soybean. In addition, it presents the same range (0-40 of the real part) of vegetation dielectric constant reported by Mavrovic et al. (2018) and Ulaby and Jedlicka (1984). However, the retrieval accuracy of SP is not evaluated due to lack of in-situ measurements. Although the estimated SP reveals a certain correlation with the area PWC index, it depends not only on the stem water content but also on the area of the leaf, height and density of vegetation.

The proposed framework may have different performance over different crop fields. Such behaviour could be attributed to the different vegetation parameters and scattering mechanisms. The dominant surface scattering component produces a strong correlation between the temporal variation of estimated and measured SM, particular over canola, wheat and winter wheat fields. However, $15 \%$ underestimation is found over winter wheat areas. This result could be partly attributed to the high area PWC, which is nearly 4500 $\mathrm{g} / \mathrm{m}^{2}$ at the beginning of the early stage. Similarly, a tendency of underestimation is found when the area PWC increases at the end of the stage.

As reported by Mattia et al. (1997), Hajnsek et al. (2009) and Jagdhuber et al. (2013), the width of $\delta$ derived from polarimetric circular coherence is strongly dependent on the surface roughness state in the case of isotropic bare surfaces. In this work, $\delta$ also shows a linear correlation with soil RMS height when it is applied to the early stage of the agricultural fields. However, their correlation weakens with the evolution of crop phenology, and the temporal variation of $\delta$ indicates a dependency on crop type and diameter (Fig. 9). 
The sensitivity of $\delta$ to crop diameter is possibly caused by the received VV-pol observations under the reflection symmetry assumption. According to Jagdhuber (2016) and Eq. (12), the circular copolarised coherence usually decreases with the increase in $S_{V V}$, which finally leads to increased $\delta$.

Regarding the diversity of crop structure and the evolution of crop phenology, this work investigates the influence of $\delta$ and co-pol phase difference on the proposed SM retrieval framework. It shows that the SM retrieval performance could be improved by using a non-zero $\Delta \varphi$ and the $\delta$ which is calculated from PolSAR data obtained on the least vegetated date. As for winter wheat, the underestimation issue is solved to some extent when $\Delta \varphi$ is set to $65^{\circ}$; for bean, canola, corn, soybean and wheat, $\Delta \varphi$ shows a slight influence on SM retrieval. This result indicates that introducing a zero $\Delta \varphi$ in SM retrieval for the randomly oriented crop areas is reasonable; however, a high $\Delta \varphi$ should be considered for vertically oriented crop fields. In other words, it suggests the need to perform an in-depth study of the double-bounce effect in vegetation.

Properly separating the volume scattering component also plays an important role in improving the performance of the proposed SM retrieval framework. Comparison of results from the multiple PolSAR observation-based SM estimation methods which use different volume scattering models indicates that the GVSM model performs better than the model of Yamaguchi et al.. However, the GVSM is not always a fully adaptive volume scattering model because the co-pol power ratio, i.e. $\gamma$, of volume scattering is possibly influenced by ground scattering.

\section{Conclusions and future perspective}

A new surface SM retrieval framework based on PD and multiple PolSAR observations is proposed in this study. We combine the extended Bragg model, the extended dihedral scattering model and the generalised volume scattering model (GVSM) to modify the PD method. Compared with traditional model-based PD methods, this framework enables the decomposition technique to be embedded with refined scattering models and can provide an analytical solution for the soil dielectric constant. In addition, similar to the eigenvaluebased decomposition technique, the unknown parameters could be solved by combining with multiple PolSAR observations and nonlinear least-squares curve fitting method. Moreover, compared with traditional decomposition models, the proposed framework does not need to determine the dominated scattering mechanism, i.e. when $\alpha$ or $\beta$ are close to -1 or 1 . 
The potential of the proposed retrieval framework is investigated by utilising the multi-incidence and multitemporal L-band UAVSAR dataset obtained from Winnipeg, Canada. The results indicate that the MIPD, MTPD, and MIMTPD algorithms provide an inversion accuracy of RMSE $<6.0 \%$ and a correlation of $R>$ 0.6 with an inversion rate of 55\%-99\%, depending on the crop type and observation date of the SMAPVEX12 campaign. The moderate correlation could be partially explained by the low dynamic change of SM during the entire period of the campaign. Although no significant difference is found between the results of the MIPD, MTPD and MIMTPD retrieval algorithms, the inversion is somewhat more accurate when using MT PolSAR observations. We also conclude that this framework could present the temporal evolution of SM better over crop fields where surface scattering dominates. Regarding the physical assumption of the multiple PolSAR configurations, we anticipate that the proposed framework will not be applicable to conditions where the surface roughness and vegetation's dielectric constant change frequently in a wide range.

The maps of crop SP are also provided with an inversion rate ranging from $40 \%$ to $99 \%$ in this work. The scatterplot of SP shows some linear relationship with crop biomass and PWC, indicateing that the derived SP has potential to describe the variation of PWC, particularly for bean, corn, soybean and wheat. In addition, the investigation of the influence of the co-pol phase difference on SM estimation indicates that the proposed SM retrieval framework can be improved with a priori knowledge of co-pol phase difference.

However, the results of the proposed framework are inevitably affected by some factors including crop phenology, PWC and surface depolarisation angle. Future work will focus on the following: (1) decreasing the influence of depolarisation angle on the retrieval framework, because the derived $\delta$ at the germination stage could be assumed to be accurate in describing surface roughness; (2) investigating the potential of the proposed framework to multi-frequency (X-, C-, and L-bands) PolSAR observations; (3) introducing the fully generalised volume scattering model and the iterative hybrid decomposition method to separate the volume scattering component in a proper way and estimate the vegetation parameters, i.e. co-pol phase difference, particle anisotropy and degree of orientation (Jagdhuber et al., 2015). In addition, the investigation of dihedral scattering effect on SM estimation can be conducted by using the ground-based SAR measurements and ground-truth data which are obtained in a controlled experimental condition.

\section{Acknowledgement}

The authors would like to thank the National Snow and Ice Data Center (NSIDC), NASA, and the SMAPVEX12 funding agencies in Canada and the United States for providing the UAVSAR datasets. The 
authors would also like to thank three anonymous reviewers for their so-called insights, and Dr. J. David Ballester-Berman for his helpful discussion and comments regarding the investigation of the influencing factors of SM retrieval framework. H. S. thanks the China Scholarship Council (CSC) for supporting his visiting at the University of Alicante, Alicante, Spain, in October 2019 - November 2020. This work was supported by the National Natural Science Foundation of China [grant numbers 61971318, 41771377, 41901286, 42071295, 41901284, U2033216]; the China Postdoctoral Science Foundation [grant number 2018M642914]. This work was supported in part by the Spanish Ministry of Science and Innovation, the State Agency of Research (AEI), and the European Funds for Regional Development (EFRD) under Project TEC2017-85244-C2-1-P.

\section{Appendix A Calculations for equations of DOI and total correlation}

In this appendix, we show that the DoI of two quad-pol SAR measurements can be calculated as follows:

$$
\begin{gathered}
D o I=N-C_{n}\left(X_{I}, X_{2}, \cdots, X_{N}\right) \\
C_{n}(X)=\frac{\sum_{i=1}^{N} H\left(X_{i}\right)-H\left(X_{I}, X_{2}, \cdots, X_{N}\right)}{H\left(X_{I}, X_{2}, \cdots, X_{N}\right)} \\
H\left(X_{I}, X_{2}, \cdots, X_{N}\right)=-\sum_{x_{1}} \cdots \sum_{x_{N}} p\left(x_{1}, x_{2}, \cdots x_{N}\right) \log _{2} p\left(x_{1}, x_{2}, \cdots x_{N}\right)
\end{gathered}
$$

where $\mathrm{N}$ is the number of variables; and $C_{n}(\cdot)$ is the normalised total correlation, which takes a value between 0 and $\mathrm{N}-1 . H\left(X_{i}\right)$ is the Shannon entropy of a single variable $X_{i} . H\left(X_{1}, X_{2}, \cdots, X_{N}\right)$ and $p\left(x_{1}, x_{2}, \cdots x_{N}\right)$ is the joint entropy and probability mass function of multiple variables, respectively (Konings et al. 2015).

\section{References}

An, Wentao, Cui, Yi, Yang, Jian, 2010. Three-component model-based decomposition for Polarimetric SAR data. IEEE Trans. Geosci. Remote Sens. 48, 2732-2739. https://doi.org/10.1109/TGRS.2010.2041242.

Antropov, O., Rauste, Y., Hame, T., 2011. Volume Scattering Modeling in PolSAR Decompositions: Study of ALOS PALSAR Data Over Boreal Forest. IEEE Trans. Geosci. Remote Sens. 49, 3838-3848. https://doi.org/10.1109/TGRS.2011.2138146

Arii, M., van Zyl, J.J., Kim, Y., 2010. A General Characterization for Polarimetric Scattering From Vegetation Canopies. IEEE Trans. Geosci. Remote Sens. 48, 3349-3357. ttps://doi.org/10.1109/TGRS.2010.2046331

Arii, M., Zyl, J.J. Van, Kim, Y., Member, S., 2011. Adaptive Model-Based Decomposition of Polarimetric SAR Covariance Matrices. Aperture 49, 1104-1113.

Chen, S.W., Wang, X.S., Xiao, S.P., Sato, M., 2014. General polarimetric model-based decomposition for 
coherency matrix. IEEE Trans. Geosci. Remote Sens. 52, 1843-1855.

https://doi.org/10.1109/TGRS.2013.2255615

Chen, Q., Zeng, J., Cui, C., Li, Z., Chen, K.-S., Bai, X., Xu, J., 2018. Soil Moisture Retrieval From SMAP: A Validation and Error Analysis Study Using Ground-Based Observations Over the Little Washita Watershed. IEEE Trans. Geosci. Remote Sens. 56, 1394-1408. https://doi.org/10.1109/TGRS.2017.2762462

Cloude, S.R., 2010. Polarisation: applications in remote sensing. Oxford University Press.

Cloude, S.R., Pottier, E., 1997. An entropy based classification scheme for land applications of polarimetric SAR. IEEE Trans. Geosci. Remote Sens. 35, 68-78. https://doi.org/10.1109/36.551935

Crow, W.T., Berg, A.A., Cosh, M.H., Loew, A., Mohanty, B.P., Panciera, R., De Rosnay, P., Ryu, D., Walker, J.P., 2012. Upscaling sparse ground-based soil moisture observations for the validation of coarseresolution satellite soil moisture products. Rev. Geophys. 50, 1-20. https://doi.org/10.1029/2011RG000372

Cui, Y., Yamaguchi, Y., Yang, J., Park, S.-E., Kobayashi, H., Singh, G., 2012. Three-Component Power Decomposition for Polarimetric SAR Data Based on Adaptive Volume Scatter Modeling. Remote Sens. 4, 1559-1572. https://doi.org/10.3390/rs4061559

Cui, Yi, Yamaguchi, Y., Yang, Jian, Kobayashi, H., Park, Sang-Eun, Singh, G., 2014. Oncomplete model-based decomposition of Polarimetric SAR coherency matrix data.IEEE Trans. Geosci. Remote Sens. 52, 19912001. https://doi.org/10.1109/TGRS.2013.2257603.

Das, N.N., Entekhabi, D., Dunbar, R.S., Chaubell, M.J., Colliander, A., Yueh, S., Jagdhuber, T., Chen, F., Crow, W., O’Neill, P.E., Walker, J.P., Berg, A., Bosch, D.D., Caldwell, T., Cosh, M.H., Collins, C.H., LopezBaeza, E., Thibeault, M., 2019. The SMAP and Copernicus Sentinel 1A/B microwave active-passive high resolution surface soil moisture product. Remote Sens. Environ. 233, 111380. https://doi.org/10.1016/j.rse.2019.111380

De Zan, F., Parizzi, A., Prats-Iraola, P., Lopez-Dekker, P., 2014. A SAR Interferometric Model for Soil Moisture. IEEE Trans. Geosci. Remote Sens. 52, 418-425. https://doi.org/10.1109/TGRS.2013.2241069

Di Martino, G., Iodice, A., Natale, A., Riccio, D., 2016. Polarimetric Two-Scale Two-Component Model for the Retrieval of Soil Moisture Under Moderate Vegetation via L-Band SAR Data. IEEE Trans. Geosci. Remote Sens. 54, 2470-2491. https://doi.org/10.1109/TGRS.2015.2502425

El Hajj, M., Baghdadi, N., Zribi, M., Belaud, G., Cheviron, B., Courault, D., Charron, F., 2016. Soil moisture retrieval over irrigated grassland using X-band SAR data. Remote Sens. Environ. 176, 202-218. https://doi.org/10.1016/j.rse.2016.01.027

Fang, B., Lakshmi, V., Jackson, T.J., Bindlish, R., Colliander, A., 2019. Passive/active microwave soil moisture change disaggregation using SMAPVEX12 data. J. Hydrol. 574, 1085-1098. https://doi.org/10.1016/j.jhydrol.2019.04.082

Freeman, A., Durden, S.L., 1998. A three-component scattering model for polarimetric SAR data. IEEE Trans. Geosci. Remote Sens. 36, 963-973. https://doi.org/10.1109/36.673687

Hajnsek, I., Jagdhuber, T., Schon, H., Papathanassiou, K.P., 2009. Potential of Estimating Soil Moisture Under Vegetation Cover by Means of PolSAR. IEEE Trans. Geosci. Remote Sens. 47, 442-454. https://doi.org/10.1109/TGRS.2008.2009642

Hajnsek, I., Pottier, E., Cloude, S.R., 2003. Inversion of surface parameters from polarimetric SAR. IEEE Trans. Geosci. Remote Sens. 41, 727-744. https://doi.org/10.1109/TGRS.2003.810702

Huang, X., Wang, J., Shang, J., 2016a. An Integrated Surface Parameter Inversion Scheme Over Agricultural Fields at Early Growing Stages by Means of C-Band Polarimetric RADARSAT-2 Imagery. IEEE Trans. Geosci. Remote Sens. 54, 2510-2528. https://doi.org/10.1109/TGRS.2015.2502600

Huang, X., Wang, J., Shang, J., 2016b. An Adaptive Two-Component Model-Based Decomposition on Soil 
Moisture Estimation for C-Band RADARSAT-2 Imagery Over Wheat Fields at Early Growing Stages. IEEE Geosci. Remote Sens. Lett. 13, 1-5. https://doi.org/10.1109/LGRS.2016.2517082

Iodice, A., Natale, A., Riccio, D., 2013. Polarimetric Two-Scale Model for Soil Moisture Retrieval via Dual-Pol HH-VV SAR Data. IEEE J. Sel. Top. Appl. Earth Obs. Remote Sens. 6, 1163-1171. https://doi.org/10.1109/JSTARS.2013.2238893

Jackson, T.J., McNairn, H., Weltz, M.A., Brisco, B., Brown, R., 1997. First order surface roughness correction of active microwave observations for estimating soil moisture. IEEE Trans. Geosci. Remote Sens. 35, 1065-1069. https://doi.org/10.1109/36.602548

Jagdhuber, T., 2012. Soil Parameter Retrieval under Vegetation Cover Using SAR Polarimetry. PhD Diss. University of Potsdam.

Jagdhuber, T., 2016. An Approach to Extended Fresnel Scattering for Modeling of Depolarizing Soil-Trunk Double-Bounce Scattering. Remote Sens. 8, 818. https://doi.org/10.3390/rs8100818

Jagdhuber, T., Hajnsek, I., Bronstert, A., Papathanassiou, K.P., 2013. Soil Moisture Estimation Under Low Vegetation Cover Using a Multi-Angular Polarimetric Decomposition. IEEE Trans. Geosci. Remote Sens. 51, 2201-2215. https://doi.org/10.1109/TGRS.2012.2209433

Jagdhuber, T., Hajnsek, I., Papathanassiou, K.P., 2015. An Iterative Generalized Hybrid Decomposition for Soil Moisture Retrieval Under Vegetation Cover Using Fully Polarimetric SAR. IEEE J. Sel. Top. Appl. Earth Obs. Remote Sens. 8, 3911-3922. https://doi.org/10.1109/JSTARS.2014.2371468

Jagdhuber, T., Baur, M., Akbar, R., Das, N.N., Link, M., He, L., Entekhabi, D., 2019. Estimation of activepassive microwave covariation using SMAP and Sentinel-1 data. Remote Sens. Environ. 225, 458-468. https://doi.org/10.1016/j.rse.2019.03.021

Karthikeyan, L., Pan, M., Wanders, N., Kumar, D.N., Wood, E.F., 2017a. Four decades of microwave satellite soil moisture observations: Part 1. A review of retrieval algorithms. Adv. Water Resour. 109, 106-120. https://doi.org/10.1016/j.advwatres.2017.09.006

Karthikeyan, L., Pan, M., Wanders, N., Kumar, D.N., Wood, E.F., 2017b. Four decades of microwave satellite soil moisture observations: Part 2. Product validation and inter-satellite comparisons. Adv. Water Resour. 109, 236-252. https://doi.org/10.1016/j.advwatres.2017.09.010

Kerr, Y.H., Waldteufel, P., Wigneron, J.-P., Delwart, S., Cabot, F., Boutin, J., Escorihuela, M.-J., Font, J., Reul, N., Gruhier, C., Juglea, S.E., Drinkwater, M.R., Hahne, A., Martín-Neira, M., Mecklenburg, S., 2010. The SMOS Mission: New Tool for Monitoring Key Elements ofthe Global Water Cycle. Proc. IEEE 98, 666687. https://doi.org/10.1109/JPROC.2010.2043032

Konings, A.G., McColl, K.A., Piles, M., Entekhabi, D., 2015. How many parameters can be maximally estimated from a set of measurements? IEEE Geosci. Remote Sens. Lett. 12, 1081-1085. https://doi.org/10.1109/LGRS.2014.2381641

Lunt, I.A., Hubbard, S.S., Rubin, Y., 2005. Soil moisture content estimation using ground-penetrating radar reflection data. J. Hydrol. 307, 254-269. https://doi.org/10.1016/j.jhydrol.2004.10.014

Mandal, D., Kumar, V., Ratha, D., Lopez-Sanchez, J.M., Bhattacharya, A., McNairn, H., Rao, Y.S., Ramana, K.V., 2020. Assessment of rice growth conditions in a semi-arid region of India using the Generalized Radar Vegetation Index derived from RADARSAT-2 polarimetric SAR data. Remote Sens. Environ. 237, 111561. https://doi.org/10.1016/j.rse.2019.111561

Mattia, F., Le Toan, T., Souyris, J.-C., De Carolis, C., Floury, N., Posa, F., Pasquariello, N.G., 1997a. The effect of surface roughness on multifrequency polarimetric SAR data. IEEE Trans. Geosci. Remote Sens. 35, 954-966. https://doi.org/10.1109/36.602537

Mavrovic, A., Roy, A., Royer, A., Filali, B., Boone, F., Pappas, C., Sonnentag, O., 2018. Dielectric characterization of vegetation at $\mathrm{L}$ band using an open-ended coaxial probe. Geosci. Instrumentation, 
Methods Data Syst. 7, 195-208. https://doi.org/10.5194/gi-7-195-2018

Mousa, B.G., Shu, H., Freeshah, M., Tariq, A., 2020. A Novel Scheme for Merging Active and Passive Satellite Soil Moisture Retrievals Based on Maximizing the Signal to Noise Ratio. Remote Sens. 12, 3804. https://doi.org/10.3390/rs12223804

Njoku, E.G., Jackson, T.J., Lakshmi, V., Chan, T.K., Nghiem, S.V., 2003. Soil moisture retrieval from AMSR-E. IEEE Trans. Geosci. Remote Sens. 41, 215-229. https://doi.org/10.1109/TGRS.2002.808243

Oh, Y., Sarabandi, K., Ulaby, F.T., 1992. An empirical model and an inversion technique for radar scattering from bare soil surfaces. IEEE Trans. Geosci. Remote Sens. 30, 370-381. https://doi.org/10.1109/36.134086

Paloscia, S., Pettinato, S., Santi, E., Notarnicola, C., Pasolli, L., Reppucci, A., 2013. Soil moisture mapping using Sentinel-1 images: Algorithm and preliminary validation. Remote Sens. Environ. 134, 234-248. https://doi.org/10.1016/j.rse.2013.02.027

Peng, J., Loew, A., Merlin, O., Verhoest, N.E.C., 2017. A review of spatial downscaling of satellite remotely sensed soil moisture. Rev. Geophys. 55, 341-366. https://doi.org/10.1002/2016RG000543

Ratha, D., Mandal, D., Kumar, V., Mcnairn, H., Bhattacharya, A., Frery, A.C., 2019. A Generalized Volume Scattering Model-Based Vegetation Index From Polarimetric SAR Data. IEEE Geosci. Remote Sens. Lett. 16, 1791-1795. https://doi.org/10.1109/LGRS.2019.2907703

Sánchez-Ruiz, S., Piles, M., Sánchez, N., Martínez-Fernández, J., Vall-llossera, M., Camps, A., 2014. Combining SMOS with visible and near/shortwave/thermal infrared satellite data for high resolution soil moisture estimates. J. Hydrol. 516, 273-283. https://doi.org/10.1016/j.jhydrol.2013.12.047

Schuler, D.L., Jong-Sen Lee, Kasilingam, D., Nesti, G., 2002. Surface roughness and slope measurements using polarimetric SAR data. IEEE Trans. Geosci. Remote Sens. 40, 687-698. https://doi.org/10.1109/TGRS.2002.1000328

Şekertekin, A., Marangoz, A.M., Abdikan, S., 2018. Soil Moisture Mapping Using Sentinel-1A Synthetic Aperture Radar Data. Int. J. Environ. Geoinformatics 5, 178-188. https://doi.org/10.30897/ijegeo.425606

Shi, H., Yang, J., Li, P., Zhao, L., Liu, Z., Zhao, J., Liu, W., 2019. Soil moisture estimation using twocomponent decomposition and a hybrid X-Bragg/Fresnel scattering model. J. Hydrol. 574, 646-659. https://doi.org/10.1016/j.jhydrol.2019.04.049

Singh, G., Yamaguchi, Y., 2018. Model-Based Six-Component Scattering Matrix Power Decomposition. IEEE Trans. Geosci. Remote Sens. 56, 5687-5704. https://doi.org/10.1109/TGRS.2018.2824322

Srikanth, P., Ramana, K. V., Deepika, U., Kalyan Chakravarthi, P., Sesha Sai, M.V.R., 2016. Comparison of Various Polarimetric Decomposition Techniques for Crop Classification. J. Indian Soc. Remote Sens. 44, 635-642. https://doi.org/10.1007/s12524-015-0525-6

Topp, G.C., Davis, J.L., Annan, A.P., 1980. Electromagnetic determination of soil water content: Measurements in coaxial transmission lines. Water Resour. Res. 16, 574-582. https://doi.org/10.1029/WR016i003p00574

Ulaby, F.T., Dubois, P.C., van Zyl, J., 1996. Radar mapping of surface soil moisture. J. Hydrol. 184, 57-84. https://doi.org/10.1016/0022-1694(95)02968-0

Ulaby, F.T., Jedlicka, R.P., 1984. Microwave Dielectric Properties of Plant Materials. IEEE Trans. Geosci. Remote Sens. GE-22, 406-415. https://doi.org/10.1109/TGRS.1984.350644

van Zyl, J.J., Arii, M., Kim, Y., 2011a. Model-Based Decomposition of Polarimetric SAR Covariance Matrices Constrained for Nonnegative Eigenvalues. IEEE Trans. Geosci. Remote Sens. 49, 3452-3459. https://doi.org/10.1109/TGRS.2011.2128325

Wang, H., Magagi, R., Goita, K., Jagdhuber, T., Hajnsek, I., 2016. Evaluation of Simplified Polarimetric Decomposition for Soil Moisture Retrieval over Vegetated Agricultural Fields. Remote Sens. 8, 142. https://doi.org/10.3390/rs8020142 
Wang, H., Magagi, R., Goita, K., 2017. Comparison of different polarimetric decompositions for soil moisture retrieval over vegetation covered agricultural area. Remote Sens. Environ. 199, 120-136. https://doi.org/10.1016/j.rse.2017.07.008

Wang, H., Magagi, R., Goïta, K., 2018. Potential of a two-component polarimetric decomposition at C-band for soil moisture retrieval over agricultural fields. Remote Sens. Environ. 217, 38-51. https://doi.org/10.1016/j.rse.2018.08.003

Wang, H., Magagi, R., Goita, K., Jagdhuber, T., 2019. Refining a Polarimetric Decomposition of Multi-Angular UAVSAR Time Series for Soil Moisture Retrieval Over Low and High Vegetated Agricultural Fields. IEEE J. Sel. Top. Appl. Earth Obs. Remote Sens. 12, 1431-1450. https://doi.org/10.1109/JSTARS.2019.2909984

Whelen, T., Siqueira, P., 2017. Use of time-series L-band UAVSAR data for the classification of agricultural fields in the San Joaquin Valley. Remote Sens. Environ. 193, 216-224. https://doi.org/10.1016/j.rse.2017.03.014

Xie, Q., Ballester-Berman, J., Lopez-Sanchez, J., Zhu, J., Wang, C., 2016. Quantitative Analysis of Polarimetric Model-Based Decomposition Methods. Remote Sens. 8, 977. https://doi.org/10.3390/rs8120977

Yamaguchi, Y., Moriyama, T., Ishido, M., Yamada, H., 2005. Four-component scattering model for polarimetric SAR image decomposition. IEEE Trans. Geosci. Remote Sens. 43, 1699-1706. https://doi.org/10.1109/TGRS.2005.852084

Yi Cui, Yamaguchi, Y., Jian Yang, Kobayashi, H., Sang-Eun Park, Singh, G., 2014. On Complete Model-Based Decomposition of Polarimetric SAR Coherency Matrix Data. IEEE Trans. Geosci. Remote Sens. 52, 1991-2001. https://doi.org/10.1109/TGRS.2013.2257603

Zebker, H., 2010. Polarisation: Applications in Remote Sensing. Phys. Today 63, 53-54. https://doi.org/10.1063/1.3502550

Zhao, L., Yang, J., Li, P., Zhang, L., 2014. Seasonal inundation monitoring and vegetation pattern mapping of the Erguna floodplain by means of a RADARSAT-2 fully polarimetric time series. Remote Sens. Environ. 152, 426-440. https://doi.org/10.1016/j.rse.2014.06.026

Zhu, L., Walker, J.P., Tsang, L., Huang, H., Ye, N., Rüdiger, C., 2019a. Soil moisture retrieval from time series multi-angular radar data using a dry down constraint. Remote Sens. Environ. 231, 111237. https://doi.org/10.1016/j.rse.2019.111237

Zhu, L., Walker, J.P., Tsang, L., Huang, H., Ye, N., Rüdiger, C., 2019b. A multi-frequency framework for soil moisture retrieval from time series radar data. Remote Sens. Environ. 235, 111433. https://doi.org/10.1016/j.rse.2019.111433

Zhuo, L., Han, D., Dai, Q., 2016. Soil moisture deficit estimation using satellite multi-angle brightness temperature. J. Hydrol. 539, 392-405. https://doi.org/10.1016/j.jhydrol.2016.05.052

Zreda, M., Shuttleworth, W.J., Zeng, X., Zweck, C., Desilets, D., Franz, T., Rosolem, R., 2012. COSMOS: the COsmic-ray Soil Moisture Observing System. Hydrol. Earth Syst. Sci. 16, 4079-4099. https://doi.org/10.5194/hess-16-4079-2012 